\title{
Report and Abstracts of the 17th Meeting of IIM, the Interuniversity Institute of Myology:Virtual meeting, October 16-18, 2020
}

\author{
Guglielmo Sorci (1), Davide Gabellini (2)
}

(1) Section of Human Anatomy, Department of Medicine \& Surgery, University of Perugia, Perugia, Italy; (2) Gene Expression and Muscular Dystrophy Group, Division of Genetics and Cell Biology, IRCCS San Raffaele Scientific Institute, Milano, Italy

This article is distributed under the terms of the Creative Commons Attribution Noncommercial License (CC BY-NC 4.0) which permits any noncommercial use, distribution, and reproduction in any medium, provided the original author(s) and source are credited.

\begin{abstract}
In 2020, due to the COVID-19 pandemic, the annual meeting of the Interuniversity Institute of Myology (IIM), took place on a virtual platform. Attendees were scientists and clinicians, as well as pharmaceutical companies and patient organization representatives from Italy, several European countries, Canada and USA. Four internationally renowned Keynote speakers presented recent advances on muscle stem cells regulation, skeletal muscle regeneration, quantitative biology approaches, and metabolic regulation of muscle homeostasis. Novel, unpublished data by young trainees were presented as oral communications or posters, in five scientific sessions and two poster sessions. On October 15, 2020, selected young trainees participated to the High Training Course on "Advanced Myology", organized together with the University of Perugia, Italy. The course, on a virtual platform, showcased lectures on muscle development and regulation of muscle gene expression by international speakers, and roundtables discussions on "Single cell analysis of skeletal muscle" and "Skeletal muscle stem cell in healthy muscle and disease". The Young IIM Committee, composed by young trainee winners of awards in the past IIM Meeting editions, was directly involved in the selection of keynote speakers, the organization of scientific sessions and roundtables discussions tailored to the interests of their peers. A broad audience of Italian, European and North American participants contributed to the different initiatives. The meeting was characterized by a friendly and inclusive atmosphere, facilitating lively and stimulating discussions on emerging areas of muscle research. The meeting stimulated scientific cross-fertilization fostering novel ideas and scientific collaborations aimed at better understanding muscle normal physiology and the mechanisms underlaying muscle diseases, with the ultimate goal of developing better therapeutic strategies. The meeting was a success, and the number of meeting attendees was the highest of all IIM Meeting editions. Despite the current difficulties imposed by the COVID-19 pandemic, we are confident that the IIM community will continue to grow and deliver significant contributions to the understanding of muscle development and function, the pathogenesis of muscular diseases and the development of novel therapeutic approaches. Here, abstracts of the meeting illustrate the new results on basic, translational, and clinical research, confirming that our field is strong and healthy.
\end{abstract}

Key Words: cachexia, clinical trials, dystrophy, epigenetics, ex-vivo, homeostasis, in-vivo, metabolism, muscle development, neuromuscular, proof of concept, regeneration, sarcopenia, stem cells, translational, wasting.

Eur J Transl Myol 2020; 30 (4), 9485. doi: 10.4081/ejtm.2020.9485

Founded in 2003 by several Italian universities, the Interuniversity Institute of Myology (IIM) has become last year a scientific association maintaining the aim to promote knowledge of the mechanisms at the basis of the physiological and pathological aspects of muscle biology, ranging from myogenesis to muscle damage, degeneration and repair, and age-related muscle atrophy. Training of young fellows and promotion of international scientific collaborations are also important goals of the association, which organizes an international IIM meeting each year. The 17th IIM Meeting (from October 16 to 18,2020 ) has been a particular edition due to the 
restrictions linked to the COVID-19 pandemic, which have forced to organize the meeting in a virtual form. Nevertheless, the meeting registered an elevated number of participants from all Europe and North America. More than 150 researchers have openly discussed together about their findings related to the pathogenesis and therapies of neuromuscular diseases, through keynote lectures, talks and poster sessions, promoting dissemination of the most recent results in the field, and suggesting novel lines of research and international collaborations. The following topics were addressed by scientists, clinicians, as well as pharmaceutical companies and patients representatives: Biophysics and E-C coupling, Genetics and epigenetics, Muscle stem cells (MuSCs) and regenerative medicine, Muscle wasting and cachexia, Exercise, Signaling and metabolism, and Therapeutic approaches. The involvement of young trainees is particularly encouraged at IIM. In line with this, the IIM Meeting 2020 has been an essential part of the 2nd edition of the university high training Course in Advanced Myology, which is the result of a collaboration between the IIM and the University of Perugia, Italy. The Course, which is reserved to people who are under 35 years old, offers the opportunity to improve knowledge in the Myology field thank to dedicated lessons and roundtable discussions with keynote international speakers in addition to the scientific contribution offered by the sessions of the meeting itself. This way, the Course in Advanced Myology represents an interesting and successful experimentation in which a scientific association supports an university in its educational activity resulting in a great-value experience particularly appreciated by young post-docs and Ph.D. students who can meet leader scientists whose studies are defining the Myology state of the art. The meeting included four Keynote Lectures by Davide Cacchiarelli (Telethon Institute of Genetics and Medicine, TIGEM, Naples, Italy), Gabrielle Kardon (University of Utah, Salt Lake City, USA), Thomas A. Rando (Stanford University, CA, USA) and Vittorio Sartorelli (National Institute of Health, Bethesda, USA). These same speakers, together with Pier Lorenzo Puri (Sanford Burnham Prebys Medical Discovery Institute, San Diego, CA, USA), were also teachers and roundtable chairs on the first day of the Advanced Myology course. Roundtable discussions were centered on "Single cell analysis of skeletal muscle: Pros and Cons" and "Skeletal muscle stem cells in healthy muscle and disease". Davide Cacchiarelli discussed the efficacy of appropriate cocktail of transcription factors to produce induced pluripotent stem cells (iPSCs) from almost any type of somatic cells, with extended self-renewal capabilities and broad differentiation potential. ${ }^{1} \mathrm{He}$ discussed the role of transcription factors during reprogramming, conversion and differentiation of human cells, and how rare genetic disorders alter the normal function of transcription factors. He also described an approach of quantitative single-cell genomics to identify the subpopulations that arise during the myogenic and pluripotency reprogramming processes, and a quantitative method to test in parallel hundreds of distinct rare variants of transcription factors associated with rare genetic diseases. Thomas A. Rando discussed mechanisms regulating MuSC quiescence and introduced the concept of G-Alert state, i.e. a particular state of quiescence that poises MuSCs to activate more rapidly and engage in tissue repair more effectively. ${ }^{2}$ He also discussed how changes in environmental signals during aging modulate the depth of quiescence of MuSCs, and the role of exercise. A potentially targetable pathway to treat the age-related decline in tissue regenerative potential was also discussed. Gabrielle Kardon discussed the property of skeletal muscle tissue to regenerate, and the key role of satellite cells in this process $^{3}$ She shared recent insights into the cellular dynamics governing satellite cell-mediated regeneration using new techniques to image the muscle regeneration process in whole-mount in combination with genetic approaches to track the fate of this cell type. She showed impressive in vivo images unrevealing the dynamics of muscle precursor cells and how myofibers are precisely reconstructed, at different times during muscle regeneration. Vittorio Sartorelli discussed the roles of enzymes and metabolites in regulating MuSC function, and how activation, proliferation and differentiation of MuSCs are energy demanding and characterized by rapidly coordinated induction of specific gene expression programs. In addition to providing energy to fuel intracellular signaling, DNA, RNA, lipid, and protein synthesis, ATP and metabolites generated by activation of defined metabolic pathways are employed by chromatin remodelers, histones and DNA modifiers to regulate gene expression. ${ }^{4}$ Pier Lorenzo Puri discussed the complexity of the 3D-genome organization in the regulation of tissue-specific gene expression, illustrating the use of chromosome conformation capture (3C)-based techniques for the analysis of the spatial organization of chromatin, and Hi-C technology for location and quantification of genomic interactions in skeletal muscles. ${ }^{5}$ In addition of providing structural and functional insights into physiological and pathological chromatin dynamics and gene expression profiles, these techniques have enabled to capture the first mapping of skeletal muscle specific high-order chromatin interactome and its relationship with muscle-specific gene expression directed by MYOD genome-wide distribution through the genome. Based on the submitted abstracts, twelve contributions were selected for Oral Communications that were subdivided into five Sessions chaired by young trainees. Fifty-two poster presentations were organized in two sessions in separated virtual rooms in order to ensure wide and fruitful discussion. Twenty amazing and very appealing poster blitzes, lasting one minute each, advised in advance the participants on posters selected by the IIM Scientific Committee. Presentations and posters provided outstanding insights 
into the Myology field, at physiological and pathological level, and offered occasions for exciting discussions involving also the young participants about extracellular factors, receptors, intracellular signaling pathways, transcription factors, and epigenetic mechanisms implicated in the regulation of cellular responses at muscle level. A virtual window was dedicated to a Parent Project association's representative, who illustrated the aim and scope of the association, its effort in sustaining the patients' families and the research, and its projects to make widely understandable the pathology of Duchenne and Becker muscular dystrophies. At the end of the meeting, special awards were assigned to the best oral communication (ex aequo to Martina Macino and Valeria Runfola), the best poster blitz (Emanuele Mocciaro) and the best poster (ex aequo to Beatrice Biferali and Gaia Gherardi), based on the evaluation by an international panel composed by IIM members. Despite its virtual form, the 17th IIM Meeting resulted very successful in putting together many myologists, from pre-clinicians to clinicians, and especially young researchers, favoring discussion, dissemination of the most recent results, exchange of ideas, formulation of new hypotheses, and international collaborations. Taken together, the attendees of the IIM and of the PaduaMuscleDays (PMDs, a meeting more oriented to advanced Translational Myology) and the authors of papers eprinted in the European Journal of Translational Myology (EJTM) are a substantial part of the international community of Myologists. ${ }^{6}$ Here, the abstracts of the 17th IIM Meeting show the relevant contribution of this community to the pathogenesis and therapies of neuromuscular diseases.

\section{List of acronyms}

EJTM - European Journal of Translational Myology

ES/iPS - satellite-like cells from human pluripotent stem cells

IMM - Interuniversity Institute of Myology

MuSC - Skeletal muscle stem cells

PMDs - PaduaMuscleDays

\section{Author's contributions}

Authors equally contributed to write the manuscript.

\section{Acknowledgements}

None

\section{Funding}

This typescript was partially sponsored by PAGEpress, Pavia, Italy and the A\&C M-C Foundation for Translational Myology, Padova, Italy.

\section{Conflict of Interest}

The authors declare they have no financial, personal, or other conflicts of interest.

\section{Ethical Publication Statement}

We confirm that we have read the Journal's position on issues involved in ethical publication and affirm that this report is consistent with those guidelines.

\section{Corresponding Authors}

Guglielmo Sorci, Section of Human Anatomy, Department of Medicine \& Surgery, University of Perugia, Perugia, Italy.

ORCID Id: 0000-0002-1973-9679

E-mail: guglielmo.sorci@unipg.it

Davide Gabellini, Gene Expression and Muscular Dystrophy Group, Division of Genetics and Cell Biology, IRCCS San Raffaele Scientific Institute, Milano, Italy. ORCID Id: 0000-0002-3811-4121

Email: gabellini.davide@hsr.it

\section{References}

1. Modic M, Cacchiarelli D, Ten Berge D. Integrative biology studies in pluripotent stem cells. Stem Cell Res 2020;42:101686. doi: 10.1016/j.scr.2019.101686

2. Biressi S, Filareto A, Rando TA Stem cell therapy for muscular dystrophies. J Clin Invest 2020;130:565264. doi: 10.1172/JCI142031

3. Agarwal M, Sharma A, Kumar P, et al. Myosin heavy chain-embryonic regulates skeletal muscle differentiation during mammalian development. Development 2020;147:dev184507. doi: 10.1242/ dev.184507

4. Sartorelli V, Lauberth SM Enhancer RNAs are an important regulatory layer of the epigenome.. Nat Struct Mol Biol 2020;27:521-8. doi: 10.1038/s41594020-0446-0

5. Dall'Agnese A, Caputo L, Nicoletti C, et al. Transcription Factor-Directed Re-wiring of Chromatin Architecture for Somatic Cell Nuclear Reprogramming toward trans-Differentiation. Mol Cell 2019;76:453-472.e8. doi: 10.1016/j.molcel.2019 .07 .036

6. Carraro U. 30 Years of Translational Mobility Medicine: 2020 Padua Muscle Days go virtual from Euganean Hills, November 19th to $21^{\text {st }}$. Eur J Transl Myol 2020;30(4):9437. doi: 10.4081/ejtm. 2020.9437

Submitted: November 11, 2020

Accepted for pubblication: November 13, 2020 


\section{$17^{\text {th }}$ IIM Meeting 16-18 October 2020}

\section{Pathogenesis and Therapies of Neuromuscular Diseases}

\section{TOPICS}

- Biophysics and E-C coupling

- Genetics and epigenetics

- Muscle stem cells and regenerative medicine

- Muscle wasting and cachexia

- Exercise

- Signaling and metabolism

- Clinical studies and therapeutic approaches

\section{Keynote Lectures}

Davide Cacchiarelli (Telethon Institute for Genetic Medicine, Napoli-Italy) Gabrielle Kardon (University of Utah, Salt Lake City-USA)

Thomas Rando (Stanford University-USA)

Vittorio Sartorelli (National Institute of Health, Bethesda-USA)

\section{Venue}

Virtual

\section{Scientific Committee}

Falcone S, Fulle S, Gabellini D, Gargioli C, Grassi F, Musarò A, Penna F, Puri PL, Sampaolesi M, Sacco A, Sorci G.

Info: https://iim2020.azuleon.org/

secretary: fisiologia@unich.it

\section{In collaboration with}




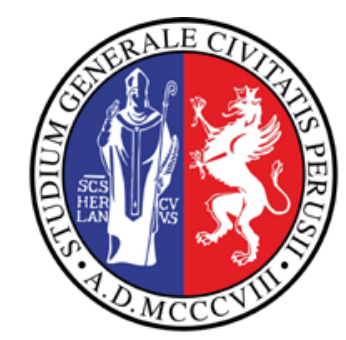

\section{UNIVERSITÀ DEGLI STUDI DI PERUGIA}

\section{Sponsored by}

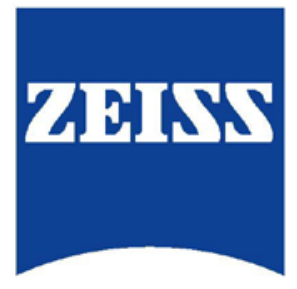

Seeing beyond

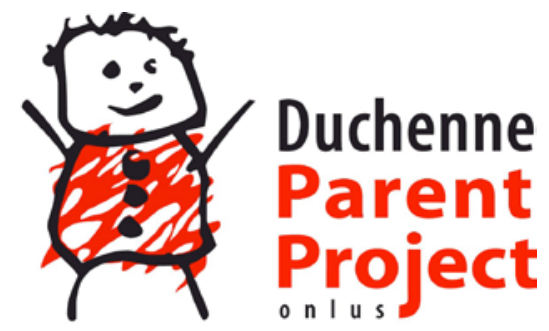




\title{
INVITED SPEAKERS
}

\section{IIM YOUNG COMMITTEE INVITED LECTURE}

\author{
Davide Cacchiarelli, PhD \\ ${ }^{1}$ TIGEM (Telethon Institute of Genetics and Medicine) - Armenise/Harvard Laboratory of \\ Integrative Genomics \\ 2University of Naples "Federico II" - Department of Translational Medicine
}

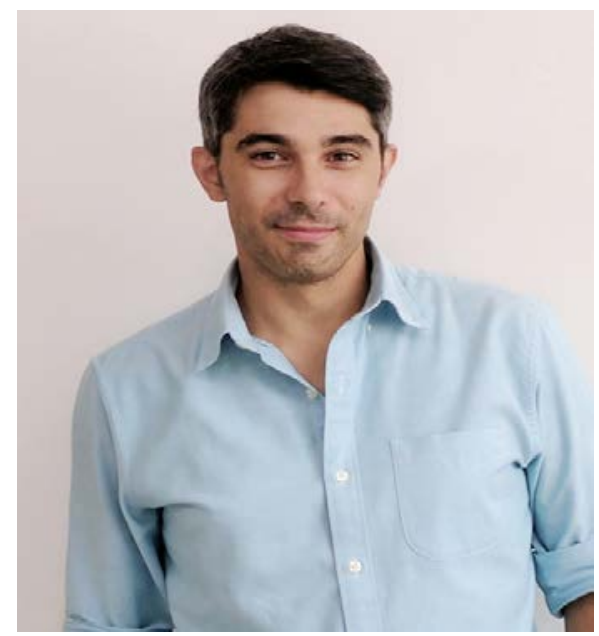

\section{Understanding transcription factors through quantitative biology}

The original concept of cell differentiation as a unidirectional process of progressively restricted potential and increased specialization has been dramatically revised by the discovery of cellular reprogramming. The appropriate cocktail of transcription factors (TFs) allows the production of Induced Pluripotent Stem Cells (IPSCs) from almost any type of somatic cell, with extended self-renewal capabilities and broad differentiation potential. This concept has infused an unprecedented boost in the use of TFs not only in reprogramming to pluripotency but, in general, to drive cell fate decisions in vitro. In our laboratory, we design and apply quantitative methods to dissect the role of transcription factors during reprogramming, conversion, and differentiation of human cells. We also study how rare genetic disorders alter the normal function of TFs (and proteins in general) with the final aim to predict the severity of rare genetic variants even before their onset in the general population. I will describe two general approaches to these scopes: i) an approach of quantitative single cell genomics to identify the subpopulations that arise during the myogenic and pluripotency reprogramming processes and reconstruct their relationships. ii) a quantitative method to test in parallel hundreds of distinct rare variants of TFs which mutations are associated with rare genetic diseases.

\section{LECTURE 2}


17th IIM Meeting (2019) - Report and Abstracts

Eur J Transl Myol 2020; 30 (4), 9485. doi: 10.4081/ejtm.2020.9485

\section{Thomas A. Rando, MD, PhD}

Professor of Neurology and Neurological Sciences

Chief, Neurology Service, Palo Alto VA Medical Center

Director, Rehabilitation Research \& Development Center of Excellence, Palo Alto VA

Medical Center

Director of the Glenn Center for the Biology of Aging at Stanford University

Deputy Director, Stanford Center on Longevity, Stanford University, CA, USA

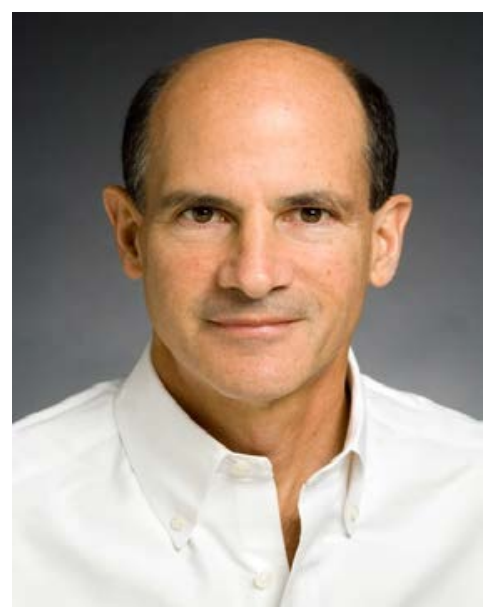

Dr. Thomas Rando received a BA from Harvard College, MD from Harvard Medical School and PhD in Cell and Developmental Biology from Harvard University Research in the Rando laboratory concerns the basic biology of stem cells how their function is altered in degenerative diseases and during aging.

Groundbreaking work from his laboratory using heterochronic parabiosis showed that the age-related changes in stem cell function can be reversed by a youthful environmental.

Dr. Rando has received many awards including an NIH Director's Pioneer Award, and he is an elected member of the National Academy of Medicine and the American Academy of Arts and Sciences.

\section{Regulation of Stem Cell Quiescence}

Many adult stem cells reside in the quiescent (G0) cell cycle state for prolonged periods of time. However, increasing evidence suggests that quiescent stem cells have active transcriptional, post-transcriptional and metabolic programs. Work from our lab and others has shown that some of these active processes serve to maintain the state of quiescence; disrupting them can lead to spontaneous activation and, in some cases, depletion of stem cells. We have also shown that quiescent stem cells respond to environmental cues that mediate dynamic transitions between different quiescent states. For example, in response to distant injuries, muscle stem cells (MuSCs) and other quiescent stem cell populations enter a state we have termed G-Alert which, while still a state of quiescence, poises the stem cells to activate more rapidly and engage in tissue repair more effectively. More recently, we have explored how changes in environmental signals during aging likewise modulate the depth of quiescence of MuSCs. Aged MuSCs are in a deeper state of quiescence. In response to exercise, aged MuSCs adopt a more poised state, albeit distinct from G-Alert. The response to exercise appears to be mediated by an induction of Cyclin D1 which, in turn, mediates its effects by acting as a transcriptional repressor for signaling pathways (such as the TGF-beta pathway) that suppress the activation of MuSCs. Inhibition of TGF-beta signaling restores the activation potential of aged MuSCs back toward a more youthful level, revealing a potentially targetable pathway to treat the age-related decline in tissue regenerative potential. 


\section{LECTURE 3}

\section{Gabrielle Kardon, PhD}

HA and Edna Benning Professor in Human Genetics

Department of Human Genetics, University of Utah, Salt Lake City, UT, USA

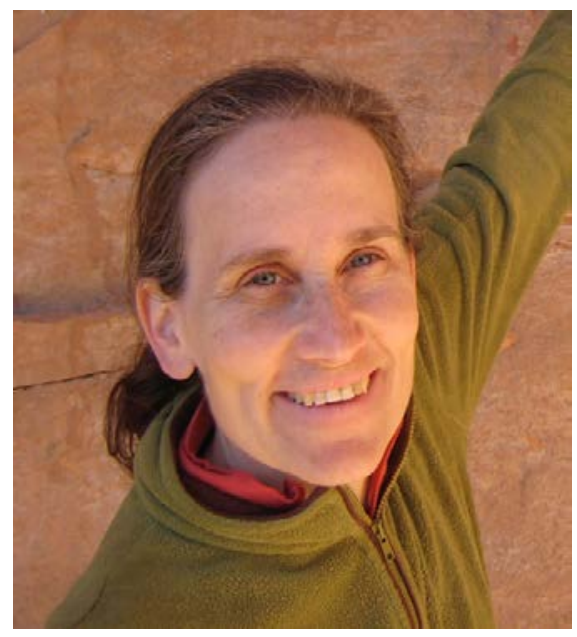

Gabrielle Kardon began her scientific career as an undergraduate at Yale University, where she majored in geology. She pursued graduate school in paleobiology at the University of Michigan and completed a MS thesis on coevolution. However, she became convinced developmental biology was her scientific path. After forays into science policy, high school teaching and world travel, she returned to academic science. She began research on the musculoskeletal system, obtaining her PhD with Dave McClay and Stephen Wainwright at Duke, and completing a postdoc with Cliff Tabin at Harvard. She started the lab in Utah in 2004. Her lab studies how interactions between muscle stem cells and connective tissue orchestrate development of limb muscles and the diaphragm, regulate muscle regeneration and aging, are the source of birth defects and fibrosis, and shape evolution of the musculoskeletal system.

\section{The Cellular Dynamics of Muscle Regeneration}

Vertebrate skeletal muscle is composed of post-mitotic, multinucleate elongated myofibers. In response to injury, these myofibers are able to be robustly regenerated. A population of dedicated stem cells, the satellite cells, is responsible for regenerating the myofibers. In the absence of injury, satellite cells are quiescent and reside in a niche between the plasmalemma and the basement of the mature myofibers. However, injury stimulates satellite cells to activate, proliferate, migrate, fuse, and differentiate and regenerate damaged myofibers. In addition, some satellite cells self-renew and re-populate the niche. Using new techniques to image muscle regeneration in whole-mount in combination with genetic lineage to track the fate of satellite cells, I will share recent insights into the cellular dynamics governing satellite cell-mediated regeneration. 


\section{LECTURE 4}

\section{Vittorio Sartorelli, MD}

Chief, Laboratory of Muscle Stem Cells and Gene Regulation

Deputy Scientific Director, National Institute of Arthritis and Musculoskeletal and Skin Diseases

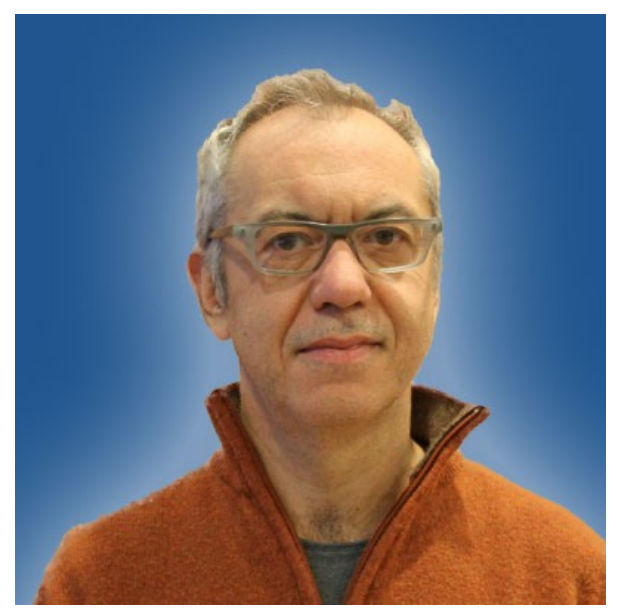

Dr. Sartorelli received his M.D. from the University of Brescia, Italy and completed a residency in oncology at the University of Milan, Italy. For his postdoc, he joined the Genetics Department and the Department of Medicine, Stanford University. Next, he joined the Department of Biochemistry and Molecular Biology, Keck School of Medicine, University of Southern California as Assistant Professor. He was then recruited to head the Muscle Gene Expression Group, Laboratory of Muscle Biology, Intramural Program at the National Institute of Arthritis and Musculoskeletal and Skin Diseases (NIAMS) within the National Institutes of Health. His laboratory focuses on the cellular and molecular mechanisms regulating specification, differentiation, and regeneration of skeletal muscle cells. The objective is to provide a conceptual and practical framework contributing to the diagnosis and treatment of human diseases affecting skeletal muscles.

\section{Enzymes and Metabolites Regulating Muscle Stem Cell Function}

Skeletal muscle growth, homeostasis, and repair rely on dedicated muscle stem cells (MuSCs). In response to mechanic stimuli or injury, otherwise quiescent MuSCs become activated, proliferate, and differentiate. These cellular processes are energetically demanding and characterized by rapidly coordinated induction of specific gene expression programs. In addition to providing energy to fuel intracellular signaling, DNA, RNA, lipid, and protein synthesis, ATP and metabolites generated by activation of defined metabolic pathways are employed by chromatin remodelers, histone and DNA modifiers to regulate gene expression. I will discuss the role of an enzyme and metabolites generated by the serine synthesis pathway in regulating MuSC function. 


\section{LECTURE 5}

\section{Pier Lorenzo Puri, MD}

Professor, Sanford Burnham Prebys Medical Discovery Institute. La Jolla, Ca.

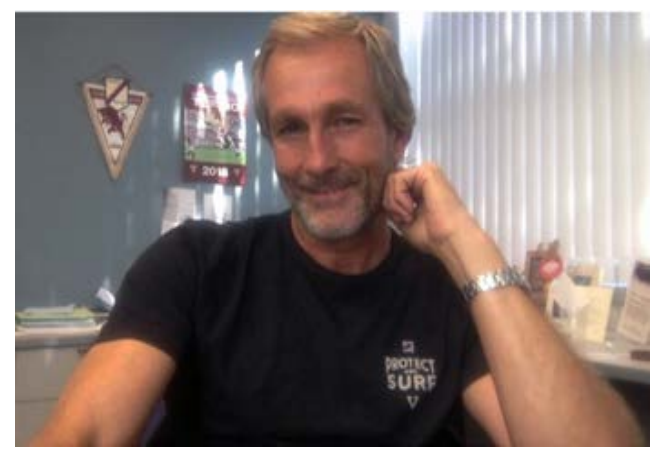

Pier Lorenzo Puri received his M.D. from the University of Roma La Sapienza and completed the internship-residency in internal medicine at the Policlinico Umberto I, Rome, Italy. He performed his postdoctoral training at the University of California San Diego. He received the first appointment as principal investigator at the Salk Institute, La Jolla, as staff scientist. Since 2004 he joined the Sanford Burnham Prebys Medical Discovery Institute, La Jolla, where he currently works as Professor. Pier Lorenzo Puri has been working as Telethon Scientist at DTI (Dulbecco Telethon Institute) from 2001 to 2012 .

\section{Molecular and epigenetic determinants of gene expression during skeletal myogenesis}

His laboratory investigates the molecular and epigenetic determinants of gene expression during skeletal myogenesis in normal conditions and in muscular dystrophies, by using multiple in vivo and ex vivo approaches. A special focus of his research in the last few years has been devoted to the understanding of the complexity of the 3D-genome organization in the regulation of tissue-specific gene expression. This interest has propelled the exploitation of chromosome conformation capture (3C)-based techniques for the analysis of the spatial organization of chromatin and $\mathrm{Hi}-\mathrm{C}$ technology for location and quantification of genomic interactions, in skeletal muscles. In addition of providing structural and functional insights into physiological and pathological chromatin dynamics and gene expression profiles, these techniques have enabled to capture the first mapping of skeletal muscle specific high-order chromatin interactome and its relationship with muscle-specific gene expression directed by MYOD genome-wide distribution through the genome. 


\section{PROGRAM SCHEDULE}

Thursday, October 15th

Program reserved to participants registered to the

High Training Course in ADVANCED MYOLOGY

16:30-19:40 High Training lectures

16.30-17.10 Lecture 1 Davide Cacchiarelli

17.10-17.20 Break

17.20-18.00 Lecture 2 Vittorio Sartorelli

18.00-18.10 Break

18.10-18.50 Lecture 3 Gabrielle Kardon

18.50-19.00 Break

19.00-19:40 Lecture 4: Pier Lorenzo Puri

19:40--20.00 Break

\section{0:00-21:00 Roundtables}

Discussants: Vittorio Sartorelli and Davide Cacchiarelli Single cell analysis of skeletal muscle: Pros and Cons Discussants: Gabrielle Kardon and Pier Lorenzo Puri

Skeletal muscle stem cell in healthy muscle and disease

Friday, October 16th

16:30-17:10 IIM young committee invited lecture

Davide Cacchiarelli (Telethon Institute for Genetic Medicine, Napoli-Italy)

Understanding transcription factors through quantitative biology.

Chair: Enrico Pozzo

17:10-17:20 Break

17:20-18:00 Lecture 2

Thomas Rando (Stanford University-USA)

Regulation of Stem Cell Quiescence.

Chair: Alessandra Sacco

18:00-18:25 Session 1. Chair: Davide Gabellini and Monica Forino The regulation of protein synthesis and muscle plasticity Ana Georgia Dumitras

Myogenin Regulates Myofibre Growth and Stem Cell Homeostasis in Adult Muscle Massimo Ganassi

18:25-18:35 POSTER BLITZ 1

3 BELTRÀ Marc

11 CZERWINSKA Areta Magda

17 FORINO Monica

19 GHERARDI Gaia

21 GIARRATANA Nefele 
27 LOMONOSOVA Yulia

37 PIN Fabrizio

39 RAITERI Tommaso

41 SALVADORI Laura

43 SERANO Matteo

18:35-18:45 Break

18:45-20:00 POSTER SESSION 1 (Odd numbers)

Saturday, October 17 th

16:30-17:10 Lecture 3:

Gabrielle Kardon (University of Utah, Salt Lake City-USA)

The Cellular Dynamics of Muscle Regeneration.

Chair: Sestina Falcone

17:10-17:20 Break

17:20-18:00 Session 2. Chair: Stefania Fulle and Gianluca Fossati

Activation of muscle-resident glial cells by nerve injury

Daisy Proietti

High Mobility Group Box 1 orchestrates regeneration in skeletal muscle

Elena Ruggieri

Identification of the first endogenous inhibitor of DUX4 in FSHD muscular dystrophy

Valeria Runfola

18:00-18:25 Session 3. Chair: Fabio Penna and Francesca Riuzzi

Decellularized skeletal muscles with neurotrophic effects for modelling 3D myogenesis in vitro

Paolo Raffa

Mechanisms and dysfunction associated to accumulation of Tubular Aggregates in aging muscle

Giorgia Rastelli

18:25-18:35 POSTER BLITZ 2

6 BIFERALI Beatrice

10 COSENTINO Marianna

16 FORCONI Flavia

24 JUPPI Hanna-Kaarina

26 LIONELLO Valentina Maria

32 MOCCIARO Emanuele

40 SACLIER Marielle

46 VAN TIENEN Florence

48 VIRLA Federica

50 YEDIGARYAN Laura

18:35-18:45 Break

18:45-20:00 POSTER SESSION 2 (Even numbers)

Sunday, October 18th

16:30-17:10 Lecture 4 
Vittorio Sartorelli (National Institute of Health, Bethesda-USA)

Enzymes and Metabolites Regulating Muscle Stem Cell Function.

Chair: Pier Lorenzo Puri

17:10-17:20 Break

17:20-18:00 Session 4. Chair: Guglielmo Sorci and Anna Urciuolo

SRF plays a major role in response to exercise in cancer patients and animal models Medhi Hassanii

Identification of two novel subpopulations of satellite cells with different kinetics of activation

Michela Libergoli

Epigenetic regulation of Wnt7b expression by the cis-acting long noncoding RNA IncRewind

in muscle stem cells

Martina Macino

18:00-18:25 Session 5. Chair: Cesare Gargioli and Letizia Zullo

High-fidelity modelling of skeletal muscle laminopathies using LMNA-mutant human iPS cells

and bioengineered muscles for mutation-specific therapy development

Luca Pinton

HDAC inhibitors tune miRNAs in extracellular vesicles of dystrophic muscle-resident mesenchymal cells

Martina Sandonà

18:25-18:35 The voice of patients

Gloria Antonini (Parent Project)

18:35-18:45 Break

18:45-20:00 Awards and prizes 


\section{POSTERS}

ODD numbers: Friday, October 16th (18:45-20:00)

EVEN numbers: Saturday, October 17th (18:45-20:00)

P. 01. Vitamin $D_{3}$ (cholecalciferol) protects skeletal muscle from cytokine-induced atrophy Maraiza Alves Teixeira

P. 02. Neuroprotective effect of epigenetic drugs in amyotrophic lateral sclerosis (ALS) murine model

Molakun Bankole

P. 03. Skeletal muscle PGC-1a overexpression impacts on progenitor cell populations Marc Beltrà

P. 04. The neurohypophyseal hormone Oxytocin is a potential anticachectic agent

Alexandra Benoni

P. 05. Altered $\mathrm{Ca}_{2+}$ dynamics and oxidative balance in GAP-43-knockout cardiomyocytes

Michele Bevere

P. 06. H3K9 methylation controls Fibro-Adipogenic Progenitors identity and skeletal muscle repair

Beatrice Biferali

P. 07. Valproic acid stimulates myogenesis in pluripotent stem cell derived-mesodermal progenitors

Natacha Breuls

P. 08. Rebalancing HMGB1 redox isoforms expression to counteract muscular dystrophy Giorgia Careccia

P. 09. Role of STAT3-mediated autophagy in driving muscle regeneration during aging

Giorgia Catarinella

P. 10. X-MET, a muscle engineered tissue for basic research and regenerative medicine Marianna Cosentino

P. 11. Fusion of blood cells to skeletal myoblasts

Areta Magda Czerwinska

P. 12. The positive modulation of the Mitochondrial Calcium Uniporter activity by Amorolfine sustains skeletal muscle trophism

Agnese De Mario

P. 13. On the impact of nuclear long noncoding RNAs in myogenesis: Charme interacts with the chromatin to fine-tune muscle specification

Fabio Desideri

P. 14. P2X7 activation enhances skeletal muscle metabolism and regeneration in SOD1G93A mouse model of Amyotrophic Lateral Sclerosis

Paola Fabbrizio

P. 15. Detrimental role of complement C1/WNT axis in dystrophic muscle

Francesca Florio

P. 16. Development and characterization of a 3D neuromuscular junction model for studying neuromuscular diseases

Flavia Forconi

P. 17. Givinostat modulates the response of TGF-beta in DMD-patient skeletal muscle

fibroblasts by inhibiting multiple HDAC enzymatic activities

Monica Forino

P. 18. Role of mTOR in skeletal muscle during cancer cachexia.

Alessia Geremia

P. 19. Mitochondrial metabolism regulates muscle homeostasis in adulthood and ageing

Gaia Gherardi

P. 20. Characterization of a DUX4 inhibitor in FSHD 
Paola Ghezzi

P. 21. Role of MICAL2 in muscle commitments and rhabdomyosarcoma progression Nefele Giarratana

P. 22. Exertional Heat Stroke: the role of external $\mathrm{Ca}_{2+}$

Barbara Girolami

P. 23. ACVR2B antagonism as a countermeasure to multi-organ perturbations in metastatic colorectal cancer cachexia

Joshua Huot

P. 24. Role of Menopausal Transition and Physical Activity in Loss of Lean and Muscle Mass: A Follow-Up Study in Middle-Aged Finnish Women

Hanna-Kaarina Juppi

P. 25. Downregulation of dynamin 2 (DNM2) rescues several forms of centronuclear and myotubular myopathies

Jocelyn Laporte

P. 26. Amphiphysin 2 (BIN1) modulates dynamin 2 in vivo and rescues dynamin 2centronuclear myopathy

Valentina Maria Lionello

P. 27. Dual role of eEF2k/eEF2 pathway in development of disuse-induced atrophy in skeletal muscle

Yulia Lomonosova

P. 28. Polyglutamine-expanded androgen receptor alters excitation-contraction coupling machinery and calcium dynamics in skeletal muscle

Caterina Marchioretti

P. 29. Allele specific silencing by RNAi of R92Q and R173W mutations in cardiac troponin T Loredana Migliore

P. 30. Gold nanoparticles for selective delivery of microRNA and drugs in dystrophic muscles

Francesco Millozzi

P. 31. Characterization of the transcription factor Nfix regulatory mechanisms in skeletal myoblasts

Giada Mura

P. 32. Identification and inhibition of a novel chromatin remodeling protein blocks DUX4 expression and rescues myogenic defects in FSHD muscular dystrophy

Emanuele Mocciaro

P. 33. Quantitative proteomics to unravel disease mechanisms and adaptive responses in MELAS and MERRF

Marta Murgia

P. 34. Trabectedin and lurbinectedin extend survival of mice bearing $\mathrm{C} 26$ colon adenocarcinoma, without affecting tumor growth or cachexia

Rosanna Piccirillo

P. 35. The discovery of Calcium Entry Units (CEUs)

Laura Pietrangelo

P. 36. Mitochondrial calcium signaling in Duchenne muscular dystrophy

Federica Placa

P. 37. RANKL blockade reduces cachexia and bone loss induced by non-metastatic cancer Fabrizio Pin

P. 38. Unraveling the use of anti-tumorigenic pro-differentiating microRNAs in pediatric rhabdomyosarcoma

Enrico Pozzo

P. 39. Investigation of the different outcomes of vitamin D supplementation to counteract skeletal muscle loss in sarcopenia and cachexia

Tommaso Raiteri

P. 40. Macrophages expressing Nfix promote the progression of Muscular Dystrophy 
Marielle Saclier

P. 41. Natural products to counteract muscle atrophy

Laura Salvadori

P. 42. Titin gene, transcripts and variants: a challenge for myologists and geneticists Marco Savarese

P. 43. High-fat diet increases the risk of environmental heatstroke in mice Matteo Serano

P. 44. Intravital 3D bioprinting

Anna Urciuolo

P. 45. Effects of a 12-week exercise intervention on muscle markers of mitochondrial function in individuals with type 1 diabetes

Giacomo Valli

P. 46. Development of the first in man clinical application of autologous mesoangioblasts in mitochondrial myopathy patients

Florence van Tienen

P. 47. A novel transgenic mouse model for arrhythmogenic cardiomyopathy

Libero Vitiello

P. 48. ASC-exosomes induce a neuroprotective effect on the SOD1(G93A) mouse model of ALS

Federica Virla

P. 49. Muscle types and their regenerative potential across animals

Letizia Zullo

P. 50. Unraveling the extracellular vesicle network to improve stem-cell based regeneration of striated muscles

Laura Yedigaryan

P. 51. Kca3.1 ion channels as a tool to protect dystrophic muscle

Marta Morotti

P. 52. Central and peripheral fatigue in Myotonic Dystrophy type 1: proposal of a clinical assessment protocol

Francesca Torri 


\section{SELECTED TALK ABSTRACTS}

\section{October 16th}

\section{SESSION 1}

\subsection{The regulation of protein synthesis and muscle plasticity}

Ana Georgia Dumitras a, Leonardo Nogara a,b, Marcus Kruger c, Bert Blaauw a,b

a. Venetian Institute of Molecular Medicine, Padua, Italy - b. Dept of biomedical sciences, Univ. of Padua, Padua, Italy - c. CECAD

Research Center, University of Cologne, Germany

The aim of this project is to identify and localize the nascent muscle proteome in vivo. The tool of choice is a transgenic mouse (MetRS) expressing a mutant methionyl-tRNA synthetase under the control of a skeletal muscle promoter. The mutated methionyl-tRNA synthetase can integrate a synthetic amino acid (ANL), instead of a methionine, in a nascent peptide chain. The synthetic amino acid side chain ends with an azide, allowing the formation of a covalent bond with an alkyne by a click chemistry reaction. The flexibility of the click reaction allows the visualization of nascent peptide chains either by western blot or immunofluorescence. By administering ANL in drinking water, we were able to identify the specific proteome synthesized during that period. The amount of labelled proteins has been assessed by WB-click and click-IHC. Surprisingly, the click IHC showed a difference in the amount of labelled proteins in different fibers. To further explore the different protein synthesis rate in different fiber types, we combined the data coming from click-IHC with a myosin fiber type staining. We observed an increase in protein synthesis in the fast-twitch oxidative fibers (2A), suggesting a correlation between protein synthesis and fiber types. All these results proved that the sensibility of the technique allows many applications, especially on pathological conditions where there is either a fiber type transition or fiber type specific loss. To see, in particular, how the protein synthesis can change in pathological conditions we crossed the Cre-MetRS mouse line with an MDX mouse model for Duchenne muscular dystrophy $(D M D)$. By using click-IHC we were able to visualize in more details protein synthesis rates in different fibers, changing as a consequence of muscle fiber damage, fibrosis and regeneration. These preliminary studies will give us the necessary tools to better understand the skeletal muscle proteome both in physiological and pathological conditions.

\subsection{Myogenin Regulates Myofibre Growth and Stem Cell Homeostasis in Adult Muscle Ganassi Massimo a, Badodi Sara b, Wanders Kees a, Zammit Peter a and Hughes Simon a a Randall Centre for Cell and Molecular Biophysics, King's College London, UK - в Blizard Institute, Barts and The London School of Medicine and Dentistry, Queen Mary University of London, UK.}

Efficient growth and maintenance of adult muscle depend on tuned activation of resident quiescent progenitors, named muscle stem cells (MuSCs) that upon activation, proliferate, and then differentiate and fuse into multinucleated myofibres. Myogenin (Myog) is an essential transcription factor orchestrating myocytes fusion during muscle development but its role in adult myogenesis is unclear. In contrast to mouse, myog-/ fish are viable, thus providing a model to study Myog role in adult muscle, which still awaits clarification. Here we explored Myog function using a newly developed method to isolate and characterise viable adult zebrafish myofibres. Crossbreeding myog-/ to pax7a:gfp background allowed ex vivo comparison of number, position and status of MuSCc from myog--and control sibling. Culture of MuSC-derived myoblasts provided time-course evaluation of gene expression, proliferation and differentiation ex vivo. Myog-ı-myofibres were smaller with a reduced number of myonuclei, leading to an increased myonuclear domain size. In contrast, the number of MuSCs per myofibre was increased, which displayed an "alerted" state, exhibiting earlier activation and faster cell-cycle entry ex vivo compared to sibling controls. Indeed, loss of Myog led to dysregulation of the mTOR pathway which dictates MuSCs activation and muscle growth, with reduced levels of fusogenic genes and 
upregulated pax7. Thus, Myogenin acts also upstream of myocytes fusion, influencing the number, positioning and quiescence of MuSCs so contributing to muscle homeostasis at multiple levels throughout life.

\subsection{Activation of muscle-resident glial cells by nerve injury}

Daisy Proietti a,b, Lorenzo Giordani c, Marco De Bardi a, Biliana Lozanoska-Ochser b, Susanna Amadio a, Cinzia Volonte a-d, Antoine Muchir c, Marina Bouche b, Alessandra Sacco e, Pier Lorenzo Puri e and Luca Madaro b

a IRCCS Fondazione Santa Lucia (FSL); Rome; Italy - b Dept AHFMO, "La Sapienza" University of Rome, Rome, Italy - c Center of Research in Myology, Sorbonne Universite, Paris, France - d CNR, Institute for Systems Analysis and Computer Science, Rome - e Development, Aging and Regeneration Program, Sanford Burnham Prebys Medical Discovery Institute, La Jolla, CA, USA.

During neurogenic muscle atrophy, the interruption of transmission of neurogenic signals to muscles, caused by loss of neuromuscular junction (NMJ) integrity, leads to muscle atrophy, an event that causes loss of muscle functionality with the obvious deleterious outcomes. NMJs degeneration is a prominent aspect of denervation, aging, and some pathological conditions, such as Amyotrophic Lateral Sclerosis (ALS). The precise molecular mechanisms and roles of muscle-resident cells, during neurogenic muscle atrophy, remain largely unknown. Evidences suggest that the effect on myofibers caused by neuron loss involves neighboring resident cells. Therefore, we analyzed the gene expression profiles at bulk and single-cell level of Itga7-expressing cells in muscle and revealed the existence of a subpopulation of muscle-resident glial cells, distinct from muscle satellite cells, that is selectively activated upon nerve injury. Upon nerve lesion, these cells expand and activate a neurotrophic program, that is turn off upon recovery of $\mathrm{NMJ}$ integrity. Moreover, these muscle-resident glial cells in ALS mouse model increase during disease progression but exhibit impaired neurotrophic activity, suggesting that defective activation of glial cells could be implicated in ALS pathogenesis. Therefore, a better understanding of the cellular and molecular signaling pathways in muscle-resident cells during traumatic or pathological denervation is critical for developing better therapies against neurogenic muscle atrophy and degenerative diseases such as ALS.

\subsection{High Mobility Group Box 1 orchestrates regeneration in skeletal muscle}

Elena Ruggieria,b, Michele Ferrara b, Giorgia Careccia b, Sara Boggio a, Ginevra Chialli a, Mario Tirone b, Andrea Gorzanelli b, Silvia Brunelli c, Marco Emilio Bianchi a,b, Emilie Venereau b.

a Vita-Salute San Raffaele University, Italy - b San Raffaele Scientific Institute, Division of Genetics and Cell Biology, Italy; c University of Milano-Bicocca, School of Medicine and Surgery, Italy.

Inflammation and tissue regeneration follow injury and consequently they are unavoidably entangled. High Mobility Group Box 1 (HMGB1) is a ubiquitous nuclear protein that is released by injured cells to serve as soluble message of tissue damage and to trigger "sterile" inflammation. We previously reported that HMGB1 promotes either inflammation or regeneration by switching among mutually exclusive redox states, and that oxidation of HMGB1 is a highly dynamic process upon acute muscle injury. HMGB1 appears to be a limiting factor in physiological conditions because $\mathrm{Hmgb1+/-} \mathrm{mice} \mathrm{show} \mathrm{a} \mathrm{marked} \mathrm{delay} \mathrm{in} \mathrm{muscle} \mathrm{repair} \mathrm{while} \mathrm{injection}$ of exogenous HMGB1 accelerates muscle regeneration. To further investigate the importance of HMGB1 in muscle regeneration, we decided to generate a whole body inducible HMGB1 KO mouse model. We observed a severe impairment in muscle regeneration in HMGB1-- mice, and more specifically a delay in leukocyte recruitment. To identify the source(s) of HMGB1 during muscle repair, we generated cell-specific HMGB1 knockout mouse models with deletion in myogenic cells, endothelial cells or platelets. We found that HMGB1 mainly derives from non-muscle cells in injured muscle. In addition, our data indicate that HMGB1 derived from different sources plays distinct roles in muscle regeneration, indicating that a timely and/or spatially regulated release of HMGB1 is required for optimal regeneration. Overall, our findings identified HMGB1 as a crucial mediator in muscle to orchestrate regeneration.

\subsection{Identification of the first endogenous inhibitor of DUX4 in FSHD muscular dystrophy} Valeria Runfola a, Maria Pannese a, Claudia Caronni a, Roberto Giambruno a, Annapaola Andolfo band Davide Gabellini a

a Division of Genetics and Cell Biology, San Raffaele Scientific Institute; s ProMiFa, Protein Microsequencing Facility, San Raffaele Scientific Institute, Milan, Italy

Facioscapulohumeral muscular dystrophy (FSHD) is the most prevalent neuromuscular disease affecting children and adults of all ages and both sexes. Unfortunately, no treatment is currently available. FSHD is caused by gain of expression of the double homeobox 4 (DUX4) gene, encoding for a transcription factor normally silent in somatic tissues. In FSHD, DUX4 activates of a pro-apoptotic program resulting in muscle 
wasting. Due to unknown molecular mechanisms, FSHD displays clinical and pathological manifestations overlapping with amyotrophic lateral sclerosis (ALS). While blocking DUX4 activity is a plausible therapeutic option for FSHD, the mechanism underlying DUX4-induced toxicity is poorly understood. We have identified MATR3 as the first direct inhibitor of DUX4. MATR3 is a nuclear protein mutated in ALS and dominant distal myopathy. We found that MATR3 blocks DUX4 expression, transcriptional function and toxicity. As a result, MATR3 administration rescues cell viability and myogenic differentiation of FSHD muscle cells while it is safe to healthy muscle cells. Notably, we characterized a short MATR3 peptide that is sufficient to directly block DUX4-induced toxicity. Our data promote MATR3 as therapeutic molecule to develop a rational treatment for FSHD, that in perspective might be applied to a spectrum of related and currently incurable diseases.

\subsection{Decellularized skeletal muscles with neurotrophic effects for modelling 3D myogenesis in vitro Paolo Raffa a, Valentina Scattolini a, Paola Caccin b, Mattia Francesco Maria Gerli c, Silvia Perin c, Paolo De Coppi c, Nicola Elvassore c-e and Anna Urciuolo a,c \\ a Institute of Pediatric Research (IRP), Fondazione Citta della Speranza - ьBiomedical Science Dept, University of Padova, Padova, Italy c University College London Great Ormond Street Institute of Child Health, London, UK - d Industrial Engineering Dept, University of Padova, Padova, Italy - eSIAIS, ShanghaiTech University, Shanghai, People's Republic of China}

Skeletal muscle is of high clinical interest since many congenital or acquired conditions can affect its function and lead to irreversible loss of tissue - volumetric muscle loss (VML). Skeletal muscle decellularization allows the generation of natural scaffolds that retain the extracellular matrix (ECM) mechanical integrity, biological activity, and three-dimensional (3D) architecture of the native tissue. Recent reports showed that in vivo implantation of decellularized muscles supports muscle regeneration in volumetric muscle loss models, including nervous system and neuromuscular junctional homing. Since the nervous system plays pivotal roles during skeletal muscle regeneration and in tissue homeostasis, support of reinnervation is a crucial aspect to be considered. However, the effect of decellularized muscles on reinnervation and on neuronal axon growth has been poorly investigated. Here we aimed to investigate neurotrophic properties exert by decellularized muscle by using 3D in vitro models to promote skeletal muscle innervation. We characterized residual protein composition of decellularized muscles by mass spectrometry and we show that scaffolds preserve structural proteins of the ECM of both skeletal muscle and peripheral nervous system. To investigate whether decellularized scaffolds could per se attract neural axons, organotypic sections of spinal cord were cultured three dimensionally in vitro, in presence or in absence of decellularized muscles. We found that neural axons extended from the spinal cord are attracted by the decellularized muscles and penetrate inside the scaffolds upon 3D coculture. In addition, our preliminary in vitro studies demonstrated the ability of decellularized muscles to support myoblasts adhesion, proliferation and differentiation. The combination of neuronal and muscular tissues within native 3D extracellular matrix represents a fascinating approach to develop 3D human skeletal muscle in vitro model able to recapitulate the physiology and architecture of the native tissue, and opens up new perspectives the treatment of clinical cases where extensive functional regeneration of the muscle is required.

3.2 Mechanisms and dysfunction associated to accumulation of Tubular Aggregates in aging muscle. Giorgia Rastelli, Laura Pietrangelo, Claudia Pecorai, Simona Boncompagni and Feliciano Protasi CAST, Center for Advanced Studies and Technology; University G. d'Annunzio of Chieti-Pescara, Chieti, Italy

Tubular aggregates (TAs), ordered arrays of sarcoplasmic reticulum (SR) tubes, form in fast twitch fibers of aged mice, preferentially in males. TAs are also the main morphological alteration found in biopsies from patients affected by TA Myopathy (TAM). TAM has been linked to mutations in the genes encoding for STIM1 and Orai1, the two proteins that mediate store-operated $\mathrm{Ca}_{2}+$ entry (SOCE), a mechanism that allows recovery of extracellular $\mathrm{Ca}_{2}+$ when the SR undergoes depletion. Our recent work, still unpublished, indicates that: i) ageing in mice (24 months old) causes STIM1 and Orai1 accumulation in TAs of EDL fibers; ii) the capability to maintain contractile force (compared to adult mice) is impaired in presence of external $\mathrm{Ca}_{2+}$ (relative force after 10 tetani: $61.6+/-3.0 \%$ in adult vs. $52.7+/-4.3 \%$ in aged EDL muscles); iii) 12 months of exercise in wheel cages for voluntary running (12-24 months of age) reduced significantly the formation of TAs (found in only $7 \mathrm{vs.} 50 \%$ of fibers). The goal of my PhD project is to improve our understandings of the mechanisms leading to accrual of TAs in skeletal muscle fibers: 1 . As TAs are never found in females, we will treat male mice (from 10 to 18 months of age) with estrogens administered in drinking water. At the end of the treatment, TAs formation will be evaluated by histology and EM, and the force of contraction in presence or absence of external $\mathrm{Ca}_{2}+$ will be evaluated by ex-vivo stimulation protocols in isolated EDL muscles. 2. As ageing is characterized by increased oxidative stress in muscle, more elevated in males that in females, we will: a. first assess levels of oxidative stress; and b. then treat mice with anti-oxidants to verify if formation of TAs is reduced. Finally, we generated 
animal models of TAM by expressing in mice a human mutation in Orai1 (G98S) and CASQ1 (D44N) found in patients affected by TAM. Preliminary results are encouraging as muscles from knockin Orai1-G98S mice develop TAs and display dysfunctional SOCE.

\begin{abstract}
4.1 SRF plays a major role in response to exercise in cancer patients and animal models. Hassani Medhi1-3, Benoni Alexandra 1-3, Gargano Caterina 2,4, Salim de Castro Gabriela 5, Chiappalupi Sara 6,3, Moresi Viviana 1,3, Adamo Sergio 1,3, Riuzzi Francesca 6,3, Sorci Guglielmo 6,3, Muscaritoli Maurizio 7, Seelaender Marilia 5,8, Sotoropoulos Athanassia 9, Xue Zhigang 2, Li Zhenlin 2, Agbulut Onnik 2, Coletti Dario $1-3$

1) Dept of Anatomical, Histological, Forensic and Orthopedic Sciences, Sapienza, University of Rome- 2) CNRS UMR 8256, INSERM ERL U1164, Biological Adaptation and Aging B2A, Sorbonne Universite, Paris, France -3) Interuniversity Institute of Myology, Italy - 4) Dept of Life Sciences, University of Modena e Reggio Emilia, Modena - 5) Dept of Cell and Tissue Biology, Institute of Biomedical Sciences, University of Sao Paulo, 05508-900 Sao Paulo, Brazil - 6) Dept of Experimental Medicine, University of Perugia, Italy - 7) Dept of Clinical Medicine, Sapienza University of Rome, Italy - 8) Dept of Surgery, Faculdade de Medicina, LIM26 HC, University of Sao Paulo - 9) Institut Cochin (Inserm U1016/ CNRS UMR8104 /UMR-S8104)
\end{abstract}

Cancer-cachexia leads to severe muscle tissue wasting which reduces patients' quality of life and survival. Physical training is used as an anti-cachexia treatment in several clinical trials, thanks to its capacity to regulate both the severe muscle wasting and the metabolic disorders which characterize cachexia. However, most of the mechanisms underlying its beneficial effects are still unknown. Serum response factor (SRF), a transcription factor having an important role in muscular growth, differentiation, and regeneration, could be essential as a mechano-transductor in response to exercise, in order to obtain the beneficial effects of exercise training in cachexia. We explored the role of SRF in tumor bearing mice with/without voluntary exercise by wheel running, as well as in cancer patients. We hypothesize that the expression and/or transcriptional activity of SRF decreases in the presence of a tumor, and that exercise can rescue it even in pathological conditions. In cachectic patients and in tumor-bearing mice, we observed a loss of body and muscle mass, a reduction of the muscle fiber cross section-area and a decreased SRF expression and transcriptional activity. Interestingly, SRF expression and activity are rescued with the practice of exercise training. Indeed, exercise rescues SRF expression in a dose-dependent manner, while also counteracting cachexia, further suggesting a link between SRF activity and exercise efficacy. We highlighted also, exercise effects are totally hampered in SRF-KO mice, demonstrating that SRF is necessary in response to exercise. In addition to the beneficial effects on muscle fibers, exercise promotes the expression of two SRF target genes of pivotal importance for the recruitment of myoblasts into the muscle fibers, i.e. IL-6 and IL-4; this observation may provide the molecular basis for the observed reduction of Pax7 expression, which is likely leading to the incorporation of myonuclei into the myofibres, further contributing to muscle homeostasis. These results prove the importance of the mechanical stimulation, induced by exercise or other means, for the regulation of muscle homeostasis, and highlight the role of SRF in transducing this stimulus into gene expression ultimately contributing to rescue muscle homeostasis in pathological conditions such as cancer cachexia.

\title{
4.2 Identification of a two novel subpopulations of satellite cells with different kinetics of activation
} Michela Libergoli a, b, Eyemen Kheir a, b, Francesca Florio a, b, Francesca Murganti a, b, Laura Vettori a, b, Marzia Belicchi c, Yvan Torrente c and Stefano Biressi a, b

a Dulbecco Telethon Institute, University of Trento; s Dept of Cellular, Computational, and Integrative Biology-CIBio, University of Trento; c Dept of Pathophysiology and Transplantation, Universita degli Studi di Milano, Fondazione IRCCS Ca' Granda Ospedale Maggiore Policlinico, Milan, Italy

Previous work from our laboratory in the mdx mouse model of Duchenne muscular dystrophy demonstrated that satellite cells (SCs) progressively acquire fibrotic features during the progression of the disease. In the process of characterizing this aberrant behavior, we serendipitously discovered that muscle SCs might be separated into two distinct subpopulations based on the expression of a mesenchymal stem cell "signature." Crucially, this separation does not correlate with the acquisition of fibroblast features; rather it separates the pool of SCs in two subpopulations, both maintaining myogenic properties in healthy muscles. These two newly identified subpopulations do not overlap with any previously reported subpopulation and may be prospectively isolated; present a different response in terms of kinetics of activation and differentiation during the regenerative process induced by acute muscle damage; show a different propensity to enter in $\mathrm{G}$ (alert) state upon distal injury; contain a different amount of mitochondria; are present in a different proportion in distinct muscle groups. Moreover, one of the two subpopulations can give rise to the other and therefore appears to be upstream in the lineage hierarchy. Importantly, although the two subpopulations of SCs are numerically similar in healthy limb muscles, one of the two subpopulations is progressively lost with time in dystrophic $m d x$ mice. Based on these data we are hypothesizing that an imbalance between the two newly identified 
subpopulations may impair regeneration in dystrophic muscles. These observations not only increase our knowledge of the molecular and cellular dynamics that are controlling normal and pathological muscle homeostasis but also open the possibility that restoring the proper functional equilibrium between subpopulations of SCs may counteract the progression of the dystrophic disease

\subsection{Epigenetic regulation of Wnt7b expression by the cis-acting long noncoding RNA Inc-Rewind in muscle stem cells \\ Martina Macino a,b, ${ }^{*}$, Andrea Cipriano a, Peruzzi c, Alessio Colantoni a, Chiara Mozzetta b,§, Monica Ballarino a,§. \\ a Dept. of Biology and Biotechnology Charles Darwin, Sapienza University of Rome- b Institute of Molecular Biology and Pathology (IBPM), National Research Council (CNR) at Sapienza University of Rome- c Center for Life Nano Science@Sapienza, Istituto Italiano di Tecnologia, Rome, Italy.* equal contribution; § correspondance.}

Skeletal muscle possesses an outstanding capacity to regenerate upon injury due to the adult muscle stem cells (MuSCs) activity. This ability requires the proper balance between MuSCs expansion and differentiation which is critical for muscle homeostasis and contributes, if deregulated, to muscle diseases. The importance of IncRNAs in the regulation of MuSCs proliferation/differentiation balance remains still poorly understood. In our laboratory, a high-throughput transcriptome screening of proliferating myoblasts and differentiating myotubes led to the identification of a subset of new polyadenylated and multi-exonic IncRNAs differentially expressed during in vitro murine myogenesis (Ballarino et al., 2015). Among them, the proliferation-specific transcripts, in particular, represent an interesting pool of novel IncRNAs that could potentially have a role in various step of MuSCs physiology. We decided to focus our attention on one candidate, the murine IncRNA transcript named as Inc-Rewind (Repressor of Wnt Induction). We found that this transcript is an abundant chromatin-associated IncRNA specifically expressed in proliferating myoblasts and MuSCs. Its knockdown strongly impairs MuSCs proliferation and expansion by influencing the expression of skeletal muscle genes and several components of the WNT (Wingless-INT) signalling pathway. Interestingly, we found the Wnt7b gene, located only $100 \mathrm{~kb}$ from Inc-Rewind locus, amongst the genes significantly upregulated upon Inc-Rewind depletion. Through a combination of different techniques, we were able to demonstrate a direct inhibitory role for Inc-Rewind on Wnt7b expression. Indeed, we found that Inc-Rewind directly interacts with the G9a histone lysine methyltransferase to regulate the specific in-cis repression of Wnt7b by deposition of the H3K9me2 epigenetic mark on its promoter. Finally, we identified a human orthologue of the murine Inc- Rewind transcript with high levels of sequence identity and with the same pattern of expression and subcellular localization as the murine counterpart. This strongly hints at the possibility of a conserved role for the transcript in human muscle processes. Overall, our findings provide novel insights into the role and importance of IncRNAs in the epigenetic regulation of adult muscle stem cells fate.

5.1 High-fidelity modelling of skeletal muscle laminopathies using LMNA-mutant human iPS cells and bioengineered muscles for mutation-specific therapy development

Luca Pinton 1,2,*, Heather Steele-Stallard 1,2,*, Daniel Moore 1,5, Shilpita Sarcar 1, Tanel Ozdemir 1,2, Sara Martina Maffioletti 1, Jean-Marie Cuisset 3, Gisele Bonne 4, Peter Steven Zammit 2,\# and Francesco Saverio Tedesco 1,5,6,\#

1 Dept of Cell and Developmental Biology, University College London, UK - 2 Randall Centre for Cell and Molecular Biophysics, King's College London, UK - 3 Centre de Reference des maladies neuromusculaires Nord/Est/lle de France, Service de Neuropediatrie, Hopital Roger Salengro, France. - 4 Sorbonne Universite, INSERM UMRS_974, Centre de Recherche en Myologie, Institut de Myologie, G.H. Pitie-Salpetriere, France - 5 The Francis Crick Institute, UK - 6 Dubowitz Neuromuscular Centre, Great Ormond Street Institute of Child Health, University College London, UK. *These authors contributed equally. \#Joint corresponding authors: F.S.T. \& P.S.Z.

Laminopathies are genetic diseases caused by mutations in the LMNA gene encoding A-type lamins. These proteins together with B-type lamins form the nuclear lamina which is responsible for maintaining nuclear/cell shape and regulating gene expression. In the skeletal muscle, mutated A-type lamins are linked to EmeryDreifuss (EDMD) and congenital muscular (CMD) dystrophies. The exact pathophysiology of laminopathies remains unknown. Additionally, the rarity of the disorder and the lack of easily accessible cell types for ex vivo studies impact on therapy development. To overcome these hurdles, we used patient-specific induced pluripotent stem cells (iPSCs) harbouring pathogenic LMNA mutations to model disease phenotypes in vitro. Three LMNA-mutant human iPSCs were differentiated into skeletal myogenic cells and myotubes. Disease associated phenotypes were observed in all genotypes, including abnormal nuclear shape and mislocalisation of nuclear lamins. Notably, complex modelling in three-dimensional artificial muscles resulted in recapitulation of nuclear abnormalities with higher fidelity than standard bi-dimensional cultures and identified nuclear length as a robust and objective outcome measure. Moreover, we generated a novel iPSC line carrying a mutation in LMNA exon 5 and directed its skeletal myogenic differentiation via a small molecule-mediated protocol. 
Notably, the resulting myotubes showed also significantly deformed (elongated) and current efforts focus on the skipping of such exon by means of antisense oligonucleotides to ameliorate the nuclear phenotype. These results demonstrate that patient-specific iPSCs can model cellular hallmarks of LMNA-related muscular dystrophies, laying the foundation for future screening platforms and gene therapies for laminopathies.

\subsection{HDAC inhibitors tune miRNAs in extracellular vesicles of dystrophic muscle-resident mesenchymalcells \\ Martina Sandona 1,2,t, Silvia Consalvi 1,t, Luca Tucciarone 1,2, Marco De Bardi 1, Manuel Scimeca 3,4,5, Daniela Francesca Angelini 1, Valentina Buffa 6, Adele D’Amico 7, Enrico Silvio Bertini 7 , Sara Cazzaniga 8, Paolo Bettica 8, Marina Bouche 2, Antonella Bongiovanni 6, Pier Lorenzo Puri 9, ${ }^{*}$ and Valentina Saccone 1,10,** \\ 1 Istituto di Ricovero e Cura a Carattere Scientifico (IRCCS), Fondazione Santa Lucia, Rome - 2 Division DAHFMO, Unit of Histology and Medical Embryology, Sapienza University of Rome- 3 Dept of Biomedicine and Prevention, University of Rome "Tor Vergata", - 4 IRCCS San Raffaele Pisana, Rome - 5 Orchidea Lab S.r.l., Rome - 6 Institute of Biomedicine and Molecular Immunology (IBIM), National Research Council (CNR) of Italy, Palermo, Italy - 7 Unit of Neuromuscular and Neurodegenerative Disorders, Bambino Gesu Children's Hospital, Rome, Italy - 8 Clinical R\&D Italfarmaco SpA, Cinisello Balsamo, Italy - 9 Development, Aging and Regeneration Program, Sanford Burnham Prebys Medical Discovery Institute, La Jolla, CA, USA - 10 Dip. Scienze della Vita e Sanita Pubblica, Universita Cattolica del Sacro Cuore, Rome, Italy ${ }^{*}$ Corresponding author ${ }^{*}$ Corresponding author. $†$ These authors contributed equally to this work}

Duchenne muscular dystrophy (DMD) is a rare neuromuscular disease characterized by cycles of degeneration/regeneration of muscles. During the progression of the disease the muscle regeneration potential is exhausted, and muscles are replaced by fibrotic scars and fat infiltration. Using the mdx mice model of $\mathrm{DMD}$, we demonstrated that the muscle regeneration is dependent by a com lex interplay of different cell types, such as Fibro-Adipogenic Progenitors (FAPs) and Muscle Stem Cell (MuS s). At early stages of disease, FAPs are able to support MuSCs differentiation, while during the progression of the disease they lose this ability and contribute to muscle degeneration leading to fat and fibrotic tissue deposition. In our recent work, we show that extracellular vesicles (EVs) released by FAPs mediate microRNA (miR) transfer to MuSCs and that exposure of dystrophic FAPs to HDAC inhibitors (HDACi) increases the intra-EV levels of a subset of miRs, which cooperatively target biological processes of therapeutic interest, including regeneration, fibrosis, and inflammation. Indeed, EVs released by FAPs of dystrophic muscles exposed to HDACi stimulate MuSC activation and expansion ex vivo, and promote regeneration, while inhibiting fibrosis and inflammation of dystrophic muscles, upon intramuscular transplantation in mdx mice, in vivo. Moreover, we observed, by inhibition of individual miRs, that miR-206 content of FAPs-derived EVs is a specific requirement for their activity on muscle regeneration of dystrophic muscles and that cooperative activity of HDACi-induced miRs is essential for the biological effect of these EVs. These data point to pharmacological modulation of EV content as novel strategy for therapeutic interventions in muscular dystrophies. 


\title{
POSTER ABSTRACTS
}

\author{
P01. Vitamin $D_{3}$ (cholecalciferol) protects skeletal muscle from cytokine-induced atrophy \\ Maraiza Alves Teixeira a, Marilisa De Feudis a, Simone Reano a, Tommaso Raiteri a, Andrea Scircoli a, F. \\ Prodam b and N. Filigheddu a \\ A Dept. of Translational Medicine, University of Piemonte Orientale, ${ }_{b}$ Dept. of Health Sciences, University of Piemonte Orientale, Italy
}

Skeletal muscle wasting may occur in several physio-pathological conditions and represents one of the main overlapping features between the physiological age-related sarcopenia and cachexia, which often associates to an underlying disease, such as cancer, especially in the erminally ill patients. We previously demonstrated that different vitamin D metab lites can have opposite effects on $\mathrm{C} 2 \mathrm{C} 12$ myotubes depending on the sites of hydroxylation 1 or doses. Assessing the effects of VD3 treatment on mouse $\mathrm{C} 2 \mathrm{C} 12$ skeletal muscle myotubes undergoing atrophy induced by IL6, we demonstrated that VD3 has a protective action, preserving $\mathrm{C} 2 \mathrm{C} 12$ myotubes size, likely through enhancing the protein synthesis and modulating autophagic flux. Cyp2r1 is the principal hepatic 25- hydroxylase responsible for the conversion of VD3 in 25(OH)VD3. Cyp2r1 lack in C2C12 myotubes, this indicates that VD3 may have a direct biological activity on the skeletal muscle. Furthermore, the protective activity of VD3 depended on the Vitamon D Receptor (VDR), suggesting that also VD3 might bind and activate VDR. In conclusion, also VD3, in addition to its best-known metabolites, may directly impact on skeletal muscle homeostasis.

1 Sustova H, De Feudis M, Reano S et al. Opposing effects of 25-hydroxy- and 1a,25-dihydroxy-vitamin $\mathrm{D}_{3}$ on pro-cachectic cytokine-and cancer conditioned medium-induced atrophy in C2C12 myotubes. Acta Physiol. 2019; 226(3):e13269.

P02. Neuroprotective effect of epigenetic drugs in Amyotrophic Lateral Sclerosis (ALS) murine model Molakun Bankole a, Ilaria Scambi a, Matilde Muccilli a, Federica Virla a, Marina Pizzi b and Raffaella Mariotti a a Department of Neurosciences, Biomedicine and Movement Sciences, University of Verona, Verona, Italy. - $b$ Department of Molecular and Translation Medicine, University of Brescia, Brescia, Italy.

ALS is a fatal neurodegenerative disease that affects motor neurons (MNs). About $20 \%$ of all familial cases of ALS are caused by mutation in Superoxide Dismutase 1 gene (SOD1). Transcriptional dysfunction which involves a defect in histone homeostasis (acetylation and deacetylation), has recently been implicated in MN degeneration. Histone homeostasis strongly depends on the activity of a family of enzymes known as histone deacetylases (HDACs). This family, which includes an important group known as sirtuins (SIRT) has been implicated in several cellular processes including cell death. Recent studies from our lab have demonstrated that the combination of two epigenetic drugs, MS-275 which inhibits HDACs, and Resveratrol, an activator of the AMP-activated kinase (AMPK)-sirtuin 1 pathway, provided neuroprotective effects and improved motor performance in SOD1 mice. However, MS-275 is currently not approved for clinical trials. Several studies have indicated that Valproate, another pharmacological inhibitor of HDACs, improves cell survival by promoting acetylation of histone, gene transcription and protein synthesis in cancer and ischemic stroke, and is currently been used in clinical trials. To improve the translational power of this approach, the overall aim of this study is to investigate the efficacy of MS-275 replacement with valproate, and explore for the first time in ALS mice models, the effect of a combination of these two epigenetic drugs, Valproate and Resveratrol, to modulate histone homeostasis and determine the capability of the epigenetic drugs to directly protect MNs from neurodegeneration in SOD1(G93A) ALS mice, improve motor performance and prolong the lifespan of ALS mice. Experiments were performed using SOD1(G93A) mice, separated into treated and conrol groups. Animals in the treated group were administered Valproate $(40 \mu \mathrm{g} / \mathrm{kg})$ and Resveratrol $(136 \mu \mathrm{g} / \mathrm{kg})$ in combination every day from post-natal day 50 until the end stage of the disease. Behavioural tests were carried out once a week to test motor function. Histological staining and stereological count of motor neurons in the lumbar tract of the spinal cord were performed to determine motor neuron survival. Immunoprecipitation and western blot was carried out to detect the acetylation of RelA protein in the lumbar tract. The intraperitoneal administration of valproate and resveratrol improved motor performance of the treated animals when compared to control group and the drug treatment significantly delayed the loss of motor function. Stereological count showed that 
the drugs protected the motor neuro s from death and a significant increase in the motor neuron number was observed in the treated group when ompared to the control. Western blot analysis also showed a restoration of RelA acetylation state. The epigenetic treatment showed to be effective in protecting lumbar spinal cord MNs of SOD1(G93A) mice as it rescued them from death and was associated with an increased number of MNs when compared to the control.

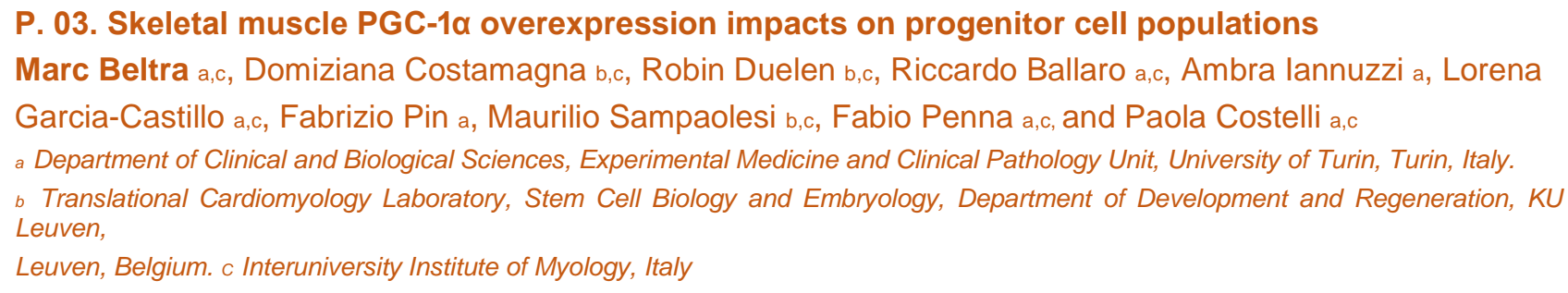

Mitochondria are key organelles in oxidative metabolism responsible for the efficient utilization of metabolic substrates necessary to produce intracellular ATP. Since mitochondria generate most of the energy required for muscle contraction, their function is directly correlated to skeletal muscle performance. The main regulator of mitochondrial biogenesis is PGC-1a, a transcriptional co-activator; its overexpression. in the skeletal muscle (MCK-PGC-1a mice) results in increased myofiber mitochondrial content1. These transgenic animals also present with an increase in the number of centrally-located nuclei in the myofibers compared to wild-type (WT) animals, indicating activation of muscle progenitors. Additionally, ex vivo experiments reveal increased expression of myosin heavy chain in myotubes obtained from MCK-PGC-1a-derived muscle progenitors compared to WT-derived ones, suggesting enhanced myotube maturation. All these evidence connect PGC$1 \alpha$ to the regulation of muscle regeneration by potentially controlling muscle progenitor activation and differentiation. Flow cytometric analysis of mononuclear cells enzymatically isolated from the skeletal muscle reveal that interstitial cells are more abundant in transgenic mice than in WT animals, including an accumulation of integrin- $\alpha 7$ positive cells (satellite cells). The increased amount of satellite cells does not tra slate into an improved regenerative capacity of the skeletal muscle after $\mathrm{BaCl}_{2}$ injury. However, cell trans lantation experiments indicate a strong propensity of MCK-PGC-1a-derived cells to fuse to myofibers f WT hosts in both healthy and damaged muscles, promoting myofiber conversion towards an oxidative ph notype. Interestingly, cell populations regarded as pro-adipogenic (CD55+cells) are reduced in PGC-1a overexpressing muscles, while negative regulators of adipocyte differentiation (Sca1+/CD142+ cells, namely Aregs2) are increased. Consistently, when n-positive areas compared to WT mice. Altogether, these results highlight PGC$1 \alpha$ regulatory role in both myogenic and non-myogenic cell populations in the skeletal muscle by potentially promoting myoblast fusion and inhibiting adipocyte differentiation.

1. Lin et al., 2002. Nature. - 2. Schwalie et al., 2018. Nature.

\section{P.04. The neurohypophyseal hormone Oxytocin is a potential anticachectic agent}

Alexandra Benoni 1,2; Medhi Hassani 1,2; Viviana Moresi 1; Zhenlin Li 2; Dario Coletti 1, 2; Zhigang Xue 2; Sergio Adamo 1

1 DAHFMO Unit of Histology and Medical Embryology, and Interuniversity Institute of Myology, Sapienza University of Rome, Italy - 2 Dept. Of Biological Adaptation and Ageing B2A (CNRS UMR 8256 - INSERM ERL U1164 - UPMC P6), Sorbonne University, France

Historically known for its effects on the uterus, the mammary gland, and the CNS, oxytocin (OT) can also affect myogenic differentiation and muscular homeostasis, since it stimulates differentiation of myogenic precursors and induces myotube hypertrophy. In addition, exogenous OT antagonizes skeletal muscle atrophy and restores skeletal muscle trophism in sarcopenic mice. Cancer cachexia has a strong negative prognostic significance in patients, being associated with a decline in quality of life and response to therapies. Several pharmacological and hormonal treatments have been proposed against cancer cachexia, but only Anamorelin (a ghrelin growth hormone secretagogue receptor agonist) is currently in phase III clinical trials. However, to date the cachexia syndrome still remains incurable. OT levels decrease in aging mice, and its exogenous administration counteracts sarcopenia. Therefore, we aim to study whether the administration of OT counteracts skeletal muscle atrophy in cancer-cachexia. To mimic in vitro cancer cell-mediated effects on muscle cells, we incubated L6 myoblasts with C26 tumor-conditioned medium (C26 CM). We observed inhibition of differentiation by $\mathrm{C} 26 \mathrm{CM}$. This effect is reversed by the addition of OT in the culture medium of 
myogenic cells both on morphological and molecular bases. We also observed in vivo, in C26-tumor-bearing mice, that daily OT injections counteract C26 dependent skeletal muscle atrophy in skeletal muscle. In addition, OT rescues muscle regeneration following focal injury, a process that is inhibited by pro-inflammatory cytokines such as TNF. Since hampered muscle regeneration and satellite cell function are important phenomena significantly contributing to muscle wasting in cachexia, our preliminary data suggest that OT treatment is effective in having a beneficial effect on muscle homeostasis in tumor bearing mice. Since OT is already approved for clinical use, these results can readily be translated into effective clinical practice to treat cancercachexia in cancer patients.

28

P.05. Altered $\mathrm{Ca}_{2+}$ dynamics and oxidative balance in GAP-43-knockout cardiomyocytes

Michele Bevere, Caterina Morabito, Maria A. Mariggio and Simone Guarnieri

Department of Neuroscience, Imaging and Clinical Sciences, Center for Advanced Studies and Technology (CAST), University "G. d'Annunzio" Chieti-Pescara, Italy

The Growth Associated Protein 43 (GAP-43) has found localized in skeletal muscle, nearby the calcium release units and, interacting with calmodulin (CaM), indirectly modulates the activities of dihydropyridine and ryanodine $\mathrm{Ca}_{2}+$ channels (Guarnieri et al, PLoS One, 2013; Caprara et al, Front Physiol, 2016). Thus, GAP$43 / \mathrm{CaM}$ interaction is one of the key player in intracellular Ca2+ homeostasis and, consequently, in the skeletal muscle activity. The control of intracellular $\mathrm{Ca}_{2}+$ signaling is also an important functional requisite in cardiac physiology. The aim of this study is to define the role of GAP-43 in intracellular $\mathrm{Ca}_{2+}$ homeostasis in cardiomyocytes and its relationship with reactive oxygen species (ROS). For this purpose, intracellular $\mathrm{Ca}_{2+}$ and ROS levels were analyzed in cardiomyocytes isolated from hearts of neonatal wild type (WT) and GAP43 knockout (KO) C57BL/6 mice. Both WT- and KO-cardiomyocytes were cultured for 7 days after isolation and then their spontaneous intracellular $\mathrm{Ca}_{2}+$ oscillations or ROS production were monitored using fluorescence video-imaging techniques (using FLUO4 and $\mathrm{H}_{2}$ DCF-DA respectively). For $\mathrm{Ca}_{2}+$ imaging, the fluorescence images were acquired at 1 frame/ $50 \mathrm{~ms}$ with a 12-bit digital EMCCD camera. The temporal analysis was expressed as $\mathrm{f} / \mathrm{fO}$ to time, where $\mathrm{f}$ is the fluorescence intensity signal in a single loaded cell that was acquired during the time-lapse, and $\mathrm{f} 0$ is the fluorescence intensity calculated from the first acquired image. Our results show that WT- and KO-cardiomyocytes presented spontaneous $\mathrm{Ca}_{2}+$ oscillations. KOcardiomyocytes showed spontaneous $\mathrm{Ca}_{2}+$ oscillations with a higher frequency and alterations in the oscillatory pattern, compared to WT-cardiomyocytes. Interestingly, KO-cardiomyocytes showed higher ROS production compared to WT-cardiomyocytes. Using specific softwares, we analyzed kinetic parameters and investigated the presence of irregularities in the $\mathrm{Ca}_{2}+$ oscillations in the presence or absence of the antioxidant $\mathrm{N}$-Acetyl- Cysteine (NAC). The administration of NAC decreased the number of cells with the abnormal rhythm in KOcardiomyocytes and did not modify the WT-cardiomyocytes activity. In conclusion, our results suggest that, in cardiomyocytes, GAP-43 may have a direct or indirect role (mediated by CaM?) in the oxidative balance and ROS production which, in turn, affect $\mathrm{Ca}_{2}+$ homeostasis.

\section{P.06. H3K9 methylation controls Fibro-Adipogenic Progenitors identity and skeletal muscle repair Beatrice Biferali a,b, Valeria Bianconi a,b, Daniel Fernandez Perez c, Sophie Pohle Kronawitter d, Fabrizia Marullo a,b, Roberta Maggio e, Tiziana Santini f, Stefano Biagionib, Vincenzo Summa g, Carlo Toniatti g, Diego Pasini c, Sigmar Stricker d, Romano Di Fabio g,h, Fulvio Chiacchiera i, Giovanna Peruzzi f, Chiara Mozzetta a. a. Institute of Molecular Biology and Pathology (IBPM), National Research Council (CNR) of Italy c/o Department of Biology and Biotechnology “C. Darwin", Sapienza University - b. Department of Biology and Biotechnology “C. Darwin”, Sapienza University, 00185 Rome, Italy - c. European Institute of Oncology (IEO), Department of Experimental Oncology, 20139, Milan, Italy - d. Institute of Chemistry and Biochemistry, Freie Universität Berlin, 14195 Berlin, Germany - e. Department of Experimental Medicine, Sapienza University of Rome (Italy) - f. Istituto Italiano di Tecnologia, Center for Life Nano Science (CLNS)@Sapienza - g. IRBM Science Park, Via Pontina Km 30.600, 00070 Pomezia, Italy - h. Promidis, Via Olgettina 60, 20132 Milano, Italy. - i. Department of Cellular, Computational and Integrative Biology (CIBIO), University of Trento, Italy}

Fibro-Adipogenic Progenitors (FAPs) are crucial regulators of muscle homeostasis as they possess the intrinsic ability to either support muscle regeneration or to contribute to fibro-adipogenic degeneration of dystrophic muscles. Therefore, the elucidation of the molecular mechanisms controlling their phenotypical 
plasticity holds therapeutic potential. Here we provide evidence that FAPs are particularly enriched in histone H3 lysine K9 methyltransferases (H3K9 KMTs), G9a, GLP and Prdm16. Our data indicate that H3K9 KMTs safeguard FAPs identity by repressing alternative transcriptional programs through deposition of $\mathrm{H} 3 \mathrm{~K} 9$ dimethylation (H3K9me2). Specifically, we show that Prdm16 controls G9a/GLP-mediated deposition of H3K9me2 at muscle-specific loci. Of note, we found Prdm16, G9a and GLP particularly enriched at the nuclear lamina (NL) of FAPs, suggesting they organize heterochromatin at the nuclear periphery to maintain the stable repression of genes encoding alternative developmental regulators. Accordingly, pharmacological inhibition or RNAi- mediated knock-down (KD) of H3K9 KMTs de-repress master myogenic genes in FAPs and induce the muscle differentiation program. Together, our findings reveal a FAPs-specific epigenetic axis important to control their identity. These findings are important especially for the possible therapeutic application to conceive strategies aimed to reprogram FAPs fate in vivo to prevent degeneration of diseased muscles.

\section{P.07. Valproic acid stimulates myogenesis in pluripotent stem cell derived-mesodermal progenitors Natacha Breuls1, Nefele Giarratana1, Laura Yedigaryan1, Paolo Carai2, Stephane Heymans2, Domiziana Costamagna1, Christophe M Deroose 3 and Maurilio Sampaolesi $1,4^{*}$ \\ 1 Laboratory of Translational Cardiomyology (Department of Development and Regeneration, Stem Cell Research Institute, KU Leuven, 3000 Leuven, Belgium) - 2 CARIM School for Cardiovascular Diseases (Department of Cardiology, Maastricht University, 6229 ER Maastricht, the Netherlands; Department of Cardiovascular Sciences, KU Leuven, 3000 Leuven, Belgium) - 3 Department of Nuclear Medicine, (University Hospital KU Leuven, Leuven, Belgium) - 4 Human Anatomy Unit (Department of Public Health, Experimental and Forensic Medicine, University of Pavia, 27100 Pavia, Italy)}

Muscular dystrophies are a group of debilitating neuromuscular disorders that affects both cardiac and skeletal muscle. For this purpose, patients would benefit from stem cells/progenitors that have the ability to regenerate both tissues. Recently, our group has successfully developed a protocol to generate mesodermal progenitors that have the ability to regenerate both cardiac and skeletal muscle (1). However, this form of cell therapy still faces many challenges. First, the current protocol lacks clinical potential as the MiPs are derived through a serum-containing embryoid body-induced differentiation protocol. Secondly, as the skeletal muscle is the largest tissue in the human body, the myogenic potential of these cells needs to be elevated. Here, we have been able to optimise a protocol to generate chemical-defined induced pluripotent stem cell-derived mesodermal progenitors (cdMiPs). These cells have the ability to contribute to the myotube formation and to differentiate into cardiomyocytes in vitro as well as in vivo. Furthermore, the addition of valproic acid (VPA) improves the myotube formation without compromising their ability to differentiate towards cardiomyocytes. This improved myogenic potential was accompanied by an increased expression of CD82+, as shown by single-cell RNA sequencing (2). Finally, the activation of the Notch signalling pathway was required for VPA to stimulate myogenesis. Taken together, we identified a novel approach to generate mesodermal progenitors and increase their myogenic potential in a translatable manner.

(1) Quattrocelli M, Swinnen M, Giacomazzi G, Camps J, Barthelemy I, Ceccarelli G, et al. Mesodermal iPSC-derived progenitor cells functionally regenerate cardiac and skeletal muscle. J Clin Invest. 2015 Dec;125(12):4463-82; (2) Camps J*, Breuls N*, Sifrim A, Giarratana N, Corvelyn M, Danti L, et al. Interstitial Cell Remodeling Promotes Aberrant Adipogenesis in Dystrophic Muscles. Cell Rep. 2020 May 5;31(5).

\section{P. 08. Rebalancing HMGB1 redox isoforms expression to counteract muscular dystrophy}

Giorgia Careccia 1,2, Marielle Saclier 3, Mario Tirone 4†, Elena Ruggieri 1,2, Elisa Principi 5, Lizzia Raffaghello 5, Silvia Torchio 3, Deborah Recchia 6, Monica Canepari 6, Andrea Gorzanelli 4, Michele Ferrara 1, Patrizia Castellani 7, Anna Rubartelli 7, Patrizia Rovere-Querini 8, Maura Casalgrandi 9, Alessandro Preti 9, Isabella Lorenzetti 10, Claudio Bruno 5, Roberto Bottinelli 6,11, Silvia Brunelli 12, Stefano Carlo Previtali 10, Marco Emilio Bianchi 1,4, Graziella Messina 3 and Emilie Venereau 1*.

1 Division of Genetics and Cell Biology, Tissue Regeneration \& Homeostasis Unit, IRCCS San Raffaele Scientific Institute; 2 San Raffaele University; з Department of Biosciences, University of Milan; 4 Division of Genetics and Cell Biology, Chromatin Dynamics Unit, IRCCS San Raffaele Scientific Institute; 5 Center of Translational and Experimental Myology, IRCCS Istituto Giannina Gaslini; 6 Department of Molecular Medicine, University of Pavia; 7 Unità di Biologia Cellulare, IRCCS Ospedale Policlinico San Martino; s Division of Immunology, Transplantation and Infectious Immunity, IRCCS Ospedale San Raffaele; , HMGBiotech srl, Milan; 10 Division of Neuroscience and Inspe, Neuromuscular Repair Unit, IRCCS San Raffaele Scientific Institute; 11 Interdepartmental Centre for the Study of Biology and Sports Medicine, University of Pavia; Fondazione Salvatore Maugeri (IRCCS), Scientific Institute of Pavia; 12 School of Medicine and Surgery, 
Muscular Dystrophies (MDs) are characterized by progressive muscle wasting associated to oxidative stress and persistent inflammation. We identified the alarmin High Mobility Group Box 1 (HMGB1) as a functional link between oxidative stress and inflammation in MDs. The oxidation of HMGB1 cysteines switches its extracellular activities from tissue regeneration to inflammation. Specifically, fully reduced HMGB1 (fr-HMGB1) supports muscle regeneration through CXCR4, by acting on both the satellite cells and their microenvironment, while the oxidized isoform called disulfide HMGB1 (ds-HMGB1) acts as proinflammatory cytokine through Toll- Like Receptor 4 (TLR4) and the Receptor for Advanced Glycation Endproducts (RAGE). We found that extracellular HMGB1 is present at high levels and undergoes oxidation in dystrophic patients and in mouse models of Duchenne Muscular Dystrophy (DMD) and Limb-Girdle Muscular Dystrophy type 2d (LGMD2D). Genetic ablation of HMGB1 in muscles of DMD mice leads to an amelioration of the dystrophic phenotype associated to decreased inflammation and muscle degeneration, while administration of ds-HMGB1 isoform rescues the dystrophic phenotype. Pharmacological treatment with a designer non-oxidizable variant of HMGB1, called 3S, improves functional performance, muscle regeneration and satellite cell engraftment in dystrophic mice, while reducing inflammation and fibrosis. Overall, our data demonstrate that the balance between HMGB1 redox isoforms dictates whether skeletal muscle is in an inflamed or regenerating state, and that the non-oxidizable form of HMGB1 is a possible therapeutic approach to counteract the progression of the dystrophic phenotype.

\section{P. 09. Role of STAT3-mediated autophagy in driving muscle regeneration during aging}

Giorgia Catarinella 1,2, Andrea Bracaglia 2,3, Elisa Bisicchia 2, Alessandra Sacco5 and Lucia Latella 2,4

1 DAHFMO, Unit of Histology and Medical Embryology, Sapienza, University of Rome, Italy; z Epigenetics and Regenerative Medicine, IRCCS Fondazione Santa Lucia, Rome, Italy; з Department of Biology, Tor Vergata, University of Rome, Italy; 4 Development, Aging and Regeneration Program, Sanford Burnham Prebys Medical Discovery Institute, La Jolla, CA, USA; 5 Institute of Translational Pharmacology,

National Research Council of Italy, Rome, Italy.

Age-related neuromuscular diseases are associated with the decline in muscle stem cell (MuSCs) function and muscle wasting. We and others have demonstrated a key role of STAT3 in regulating MuSCs expansion and differentiation. In parallel, we recently described the essential role of autophagy in driving MuSCs function toward efficient muscle regeneration. The well known role of autophagy in maintaining muscle mass and tissue homeostasis together with the emerging role of STAT3 in regulating the autophagic process inspired the rationale behind this project which resides in the study of the STAT3-mediated autophagy toward skeletal muscle repair. Our hypothesis is that STAT3 might have a role in regulating myogenic lineage and regeneration process by affecting the autophagic process thereby restoring the bioenergetic demand of the myogenic niche to support muscle regeneration. We show that STAT3 inhibitor (STAT3i) treatment induces the autophagic process upon muscle regeneration both in vitro and in vivo, a function that conceivably is influenced by STAT3 nucleus/cytoplasmic localization. First we show that STAT3i treatment promotes the autophagic process during muscle regeneration and this is associated with elF2! phosphorylation. In this scenario the phosphorylation of elF2! is achieved by PKR that is no longer sequestered by STAT3. Interestingly, STAT3i treatment resumes the autophagic process in old mice, otherwise characterized by low autophagy and cytoplasmic STAT3. This is coupled with the STAT3 imediated positive impact in muscle regeneration. Altogether, these evidences suggest the importance of STAT3 nuclear/cytoplasmic compartmentalization in regulating autophagy and the regenerative drive, highlighting potential biological targets that may restart an efficient regenerative response in aged mice.

\section{P. 10. X-MET, a muscle engineered tissue for basic research and regenerative medicine}

Marianna Cosentino a, Carmine Nicoletti a, Francesca Martelli b, Emanuele Rizzuto b, Zaccaria del Prete b and

Antonio Musarò a

a DAHFMO-Unit of Histology and Medical Embryology, s Department of Mechanical and Aerospace Engineering, University of Rome "La

Sapienza", Italy 
Engineered tissue is a promising therapeutic approach for enhancing tissue revascularization and regeneration. The $\mathrm{X}-\mathrm{MET}$ is a muscle engineered tissue obtained through in vitro culture of murine stem cells and realized without the use of any scaffold (Carosio et al., 2013). The X-MET displays several morphofunctional and biomechanical properties of an in vivo muscle, it is able to contract spontaneously as well as to respond to electrical stimulation (Pisu et al., 2019). The aim of our study is to define the functional plasticity of X-MET subjected to mechanical stimuli. In this work we demonstrate that different mechanical tensions can induce a functional remodelling of X-MET. Specifically, X-MET shows preserved cellular communication junctions, endogenous extracellular matrix (ECM), and integrative adhesive agents that could create a favorable environment for survival and integration of the X-MET in the host tissue. For these features, X-MET can be considered a useful experimental tool for in vitro and in vivo studies to analyze homeostatic processes in a more complex in vitro model, for drug screening and in regenerative medicine to restore the loss of muscle function.

\section{P. 11. Fusion of blood cells to skeletal myoblasts \\ Laura Galvis a, Areta Magda Czerwinska a,b, Lina Wang a,c, Christophe Marcelle a \\ a Institute NeuroMyoGene, Université Claude Bernard Lyon 1, France - в Department of Cytology, Faculty of Biology, University of Warsaw, Poland - c Australian Regenerative Medicine Institute, Monash University, Australia}

Skeletal muscles are characterized by an exceptional capacity to regenerate due to the adult muscle stem cells, named satellite cells. Satellite cells are extremely efficient at repairing damaged muscles but their use in regenerative medicine is hardly possible due to their poor engraftment and survival after injection into diseased muscles. Duchenne's muscular dystrophy (DMD) is among severe and devastating diseases that, despite many years of investigation, are still incurable. The cause for DMD is a mutation in gene encoding dystrophin, a protein crucial for anchorage of the contracting apparatus to the myofiber membrane. All cell therapies that have been tested so far for the treatment of DMD proved to be disappointing. Among the obstacles to use of grafted cells for treatment of dystrophies is the lack of a method to deliver them systemically to all affected muscles. The aim of our study was to test if blood cells, which naturally circulate in cardiovascular system, can be induced to fuse with chicken skeletal myoblasts. For this aim we have modified various cell types to express two master fusion genes, i.e. Myomaker and Myomixer. We have also developed a method to quantify heterologous fusion efficiency. As a result, we have proven different potential of various cell types expressing the same vectors, for fusion with skeletal myoblasts. Concluding, we have proven that fusion of appropriately modified immune cells to skeletal muscle myoblasts is possible.

\section{P. 12. The positive modulation of the Mitochondrial Calcium Uniporter activity by Amorolfine sustains skeletal muscle trophism \\ Agnese De Mario a, Anna Tosatto a, Gyorgy Szabadkai a,b, Rosario Rizzuto a and Cristina Mammucari a a Department of Biomedical Sciences, University of Padua, Padua, Italy - $b$ Department of Cell and Developmental Biology, Consortium for Mitochondrial Research, University College London, London, UK}

Mitochondria represent fundamental checkpoints for muscle homeostasis, since they are a major source of ATP production, thus sustaining muscle activity and functions. Skeletal muscle mitochondria readily accumulate $\mathrm{Ca}_{2+}$ in response to SR store-releasing stimuli thanks to the activity of the mitochondrial calcium uniporter (MCU), the highly selective channel responsible for mitochondrial $\mathrm{Ca}_{2+}$ (mitCa2+) uptake. In skeletal muscle, MCU-dependent mitCa2+ positively regulates myofiber size by impinging on PGC1a4 and IGF1AKT/PKB pathways. While the genetic modulation of the MCU has been widely applied, small molecules able to increase mitCa2+ uptake are rare. By using a well-established methodology based on Aequorin, a calcium sensitive probe that emits light upon $\mathrm{Ca}_{2}$ - binding, we screened a library of 1,600 FDA-approved drugs for their ability to modulate mitCa2+ uptake in living cells. We identified Amorolfine as a positive MCU modulator. Amorolfine is a morpholine antifungal drug that inhibits enzymes of the fungal sterol synthesis pathway and it is indicated for the topic treatment of mycoses. Amorolfine increases mitCa2+ uptake in Hela, $\mathrm{C} 2 \mathrm{C} 12 \mathrm{cells}$ and adult isolated myofibers without affecting cytCa2+ and mitochondrial membrane potential. In agreement with the role of $\mathrm{MCU}$ in triggering hypertrophy, Amorolfine increases the size of $\mathrm{C} 2 \mathrm{C} 12$ myotubes in an MCUdependent manner, and triggers muscle hypertrophy in vivo. Thus, these data indicate that Amorolfine, by modulating $\mathrm{MCU}$, positively regulates muscle trophism. In the future, we aim to verify whether Amorolfine treatment could exert a protective effect against skeletal muscle atrophy and sarcopenia. 


\begin{abstract}
P. 13. On the impact of nuclear long noncoding RNAs in myogenesis: Charme interacts with the chromatin to fine-tune muscle specification

Fabio Desideri 2, Andrea Cipriano 3, Giulia Buonaiuto 1, Tiziana Santini 2, Valeria Taliani 1, Silvia Petrezselyova 4, Alessio Colantoni 1, Carmine Nicoletti 5, Antonio Musaro 5, Irene Bozzoni 1,2, Monica Ballarino 1

1Department of Biology and Biotechnology "Charles Darwin" Sapienza University of Rome- 2 Center for life Nano Science @Sapienza, Istituto Italiano di Tecnologia, Rome, Italy - 3 Department of Obstetrics \& Gynecology, Stanford University, USA; 4 Czech Centre of Phenogenomics and Laboratory of Transgenic Models of Diseases, Institute of Molecular Genetics of the Czech Academy of Sciences, Vestec, Czech Republic - 5 DAHFMO-Unit of Histology and Medical Embryology, Sapienza University of Rome, Via A. Scarpa, 14, 00161 , Rome, Italy
\end{abstract}

The mammalian genome contains thousands of long noncoding RNAs (IncRNAs), which have been proposed to be fundamental in the regulation of many biological processes. Among them, nuclear IncRNAs are generally associated to chromatin and they can act as genome architects contributing to the formation or disassembly of 3D structures. Through an high-throughput transcriptome screening, we identified a subset of new polyadenylated and multiexonic IncRNAs which result differentially expressed during murine skeletal muscle in vitro differentiation (Ballarino et al., 2015). In particular one candidate, Charme (for Chromatin architect of muscle expression) is an abundant and highly conserved noncoding transcript specifically required for in vitro myogenesis. Interestingly, Charme ablation in vivo resulted in a very distinct cardiac pathological phenotype in which the morphology of the murine heart is remodelled (Ballarino et al., 2018). Mechanistically, Charme acts in the nucleus as a structural RNA, contributing to the formation of chromosome territories where coordinated expression of pro-myogenic genes occurs. Our recent finding, that intronic determinants drive the interaction between Charme and components of the nuclear matrix, adds another tier in the intriguing connection between Charme expression and chromatin shaping. New data on the need of such RNA-protein interplay for the acquisition of muscle identities both in vitro and in vivo will be presented.

\title{
P. 14. P2X7 activation enhances skeletal muscle metabolism and regeneration in SOD1G93A mouse model of Amyotrophic Lateral Sclerosis \\ Paola Fabbrizio a, Savina Apolloni b, Andrea Bianchi b, Illari Salvatori b, Cristiana Valle b,c, Chiara Lanzuolo b,d, Cinzia Volonte b,e, Caterina Bendotti a and Giovanni Nardo a \\ a Laboratory of Molecular Neurobiology, Department of Neuroscience, Istituto di Ricerche Farmacologiche Mario Negri IRCCS Milan - ${ }_{b}$ IRCCS Fondazione Santa Lucia, Rome - c National Research Council, Institute of Translational Pharmacology, Rome - dNational Research Council, Institute of Biomedical Technologies, Milan - eNational Research Council, Institute for Systems Analysis and Computer Science, Rome, Italy
}

Muscle weakness plays an important role in neuromuscular disorders comprising amyotrophic lateral sclerosis (ALS). However, it is not established whether muscle denervation originates from the motor neurons, the muscles or more likely both. Previous studies have shown that the expression of the SOD1G93A mutation in skeletal muscles causes denervation of the neuromuscular junctions, inability to regenerate and consequent atrophy, all clear symptoms of ALS. In this work, we used SOD1G93A mice, a model that best mimics some pathological features of both familial and sporadic ALS, and we investigated some biological effects induced by the activation of the $\mathrm{P} 2 \mathrm{X7}$ receptor in the skeletal muscles. The P2X7, belonging to the ionotropic family of purinergic receptors for extracellular ATP, is abundantly expressed in the healthy skeletal muscles, where it controls cell duplication, differentiation, regeneration or death. In particular, we evaluated whether an in vivo treatment in SOD1G93A mice with the P2X7 specific agonist 2'(3')-O-(4-Benzoylbenzoyl) adenosine 5'triphosphate (BzATP) just before the onset of a pathological neuromuscular phenotype, could exert beneficial effects in the skeletal muscles. Our findings indicate that stimulation of P2X7 improves the innervation and metabolism of myofibers, moreover elicits the proliferation/differentiation of satellite cells, thus preventing the denervation atrophy of skeletal muscles in SOD1G93A mice. Overall, this study suggests that a P2X7-targeted and site-specific modulation might be a strategy to interfere with the complex multifactorial and multisystem nature of ALS.

\section{P. 15. Detrimental role of complement C1/WNT axis in dystrophic muscle \\ Francesca Florio a,b, Filomena Papa a,b, Michela Libergoli a,b, Silvia Accordini a,b, Sara Vencato a,b, Imen}


17th IIM Meeting (2019) - Report and Abstracts

Eur J Transl Myol 2020; 30 (4), 9485. doi: 10.4081/ejtm.2020.9485

Ghzaiel a,b, Vaibhav Gharat a,b, Thomas A. Rando c,d, Stefano Biressi a,b

a Dulbecco Telethon Institute, University of Trento - s Department of Cellular, Computational, and Integrative Biology (CIBio), University of Trento, Trento, Italy - c Department of Neurology and Neurological Sciences and Paul F. Glenn Center for the Biology of Aging, Stanford University School of Medicine, Stanford, CA, USA - $d$ Center for Tissue Regeneration, Repair and Restoration, Veterans Affairs Palo Alto Health Care System, Palo Alto, CA 4, USA

Duchenne muscular dystrophy (DMD) is the most common inherited muscle disease of childhood. Although under normal conditions muscle stem cells present a very powerful regenerative response, the regenerative potential is progressively lost in patients affected by DMD and fibrotic tissue progressively replaces muscle fibers leading to an impairment of muscle function. Recent observations from different laboratories, including our, are indicating that an elevated WNT-signaling in dystrophic muscles plays a detrimental role in the regenerative process and promotes the accumulation of fibrotic tissue. Still, the molecular and cellular events responsible for the elevated WNT-signaling in dystrophic muscle are completely unexplored. Importantly, in addition to the role in innate immunity, the complement complex C1q has been recently reported to activate the canonical WNT-signaling. Based on these observations we formulated the hypothesis that complement C1 complex could be responsible for the enhanced activity of the WNT-signaling pathway in DMD. By combining in vitro and in vivo approaches, we obtained compelling evidence supporting this idea. In particular, our observations indicate that cells colonizing the muscles of the dystrophic mdx mice can secrete distinct subunits of the complement complex $\mathrm{C} 1$ and can therefore act as combinatorial source of WNT-activity.

\section{P. 16. Development and characterization of a 3D neuromuscular junction model for studying neuromuscular diseases \\ Flavia Forconi a, Marianna Cosentino a, Emanuele Rizzuto b and Antonio Musarò a a DAHFMO-Unit of Histology and Medical Embryology, University of Rome La Sapienza - $b$ Department of Mechanical and Aerospace Engineering, University of Rome La Sapienza, Italy}

The neuromuscular junction (NMJ) is a synaptic structure that plays a pivotal role in exchanging signals among lower motor neurons (LMNs) and skeletal muscles fibers (Das M. et al., 2010). Investigating the functionality of neuron conduction and muscle contraction is crucial in all those pathological conditions where NMJ is altered, such as Amyotrophic Lateral Sclerosis (ALS) and aging. Despite the NMJ has been widely investigated in animal models of neurodegenerative and muscular disorders (Fogarty M. J. et al., 2019), the mechanism behind the pathophysiology of these pathologies still needs to be clarified. Indeed, the development of a NMJ engineered model may represent an innovative tool to help pointing out the synaptic transmission alterations. In a previous work, we already proposed the generation of an in-vitro bioengineered three-dimensional vascularized skeletal muscle tissue (X-MET), able to recapitulate the architecture and the function of an invivo muscle (Carosio et al., 2013). On this basis, in this project we aim at developing a 3D model of neuromuscular junction, by using the X-MET and motor neuron primary culture. This model is expected to allow improving the investigation of NMJ functionality in several pathological conditions. To do this, we will initially characterize the bi-dimensional interaction between mouse spinal cord primary cultures and primary cultures obtained using muscle tissue harvested from mouse hind limbs, through a commercial 2D micro-fluidic device for NMJ formation. Subsequently, a new 3D micro-fluidic device will be projected and developed to promote the formation of synaptic transmission between mouse spinal cord primary cultures and the X-MET. Finally, the 3D NMJ model will be deeply characterized from a functional and molecular point of view.

\section{P. 17. Givinostat modulates the response of TGF-b in DMD-patient skeletal muscle fibroblasts by inhibiting multiple HDAC enzymatic activities \\ Monica Forino, Valeria Spadotto, Christian Steinkuhler and Gianluca Fossati \\ Italfarmaco SpA, New Drug Incubator Department, Italy}

Lack of dystrophin in Duchenne Muscular Dystrophy (DMD) leads to degeneration of muscle fibers progressively substituted by fibrous tissue. In DMD, TGF-b has a recognized pro-fibrotic role and levels of TGF-b are elevated in plasma and in muscles of patients and correlate with increased fibrosis. In vitro, TGF-b is highly expressed in DMD myotubes and in muscle-derived fibroblasts. Inactive TGF-b is bound to the latent TGF-b binding protein (LTBP) and in DMD patients, four LTBP4 SNPs are predictive of the age at onset of 
loss of ambulation. The LTBP4 IAAM variant releases less TGF-b and is associated with older age at loss of ambulation and less TGF- $\beta$ signaling. Histone deacetylases (HDACs) are involved in fibrogenesis and reduction of HDAC activities hinders TGF-b induced fibrosis. Our HDAC inhibitor givinostat significantly reduced fibrosis in mdx mice and outstandingly, in DMD patients. Here, we described the effects of givinostat on TGF-b induced pro-fibrotic response in fibroblasts isolated from skeletal muscle of a DMD-patient. Givinostat counteracted the effects of TGF-b by acting at two complementary levels: the reduction of the expression of pro-fibrotic genes, such as collagen I and III, and the induction of anti-fibrotic genes like PGC1a. A different regulation of genes belonging to canonical and non-canonical TGF- $\beta$ pathways also occurred. TGF R1 was upregulated by the cytokine and downregulated by givinostat while TGF R2 was downmodulated by TGF- $\beta$ and upregulated by givinostat. The upregulation of NF-kB, induced by TGF-b, was decreased to basal level by givinostat. Increased acetylation of histones and tubulin indicated target engagement by the inhibitor. Furthermore, givinostat acted at a further level of epigenetic regulation by inducing histone crotonylation, in agrement with the recently described de-crotonylase activity of class I HDACs. These data emphasize how multiple epigenetic regulations of key fibrotic genes could takes place in DMD-patient cells treated with a HDAC inhibitor. They also highlight the importance of having an in vitro model to study the specific pathways that can be targeted in diseased cells to more accurately advance drug candidates into in vivo studies.

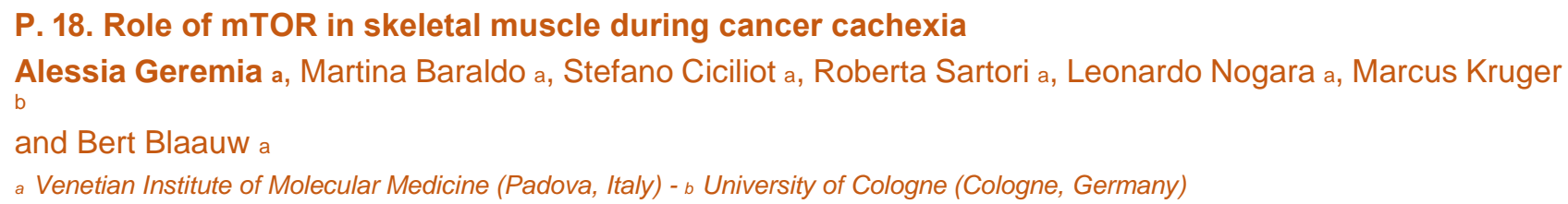

Cancer cachexia is a multi-organ syndrome which is characterized by a major loss in body weight, particularly in muscle and adipose tissue. It has been shown that if this cancer-related muscle wasting is reserved 0 prevented, lifespan is significantly improved. A critical mediator of adult muscle mass and function in skeletal muscle is the kinase mTOR, however its role during cancer cachexia is unknown yet. To assess the function of mTOR during cancer cachexia we used two loss of function mouse models in which we have deleted either mTOR, or the core mTOR complex 1 scaffold protein Raptor, only in adult skeletal muscle. Using different models of cancer cachexia we observe an impairment in autophagy and an increase in denervation markers in ko tumor-bearing mice. Interestingly, preliminary results show that activation of mTOR signaling is sufficient to restore muscle wasting during cancer cachexia, underlining its importance in cachexia dependent muscle wasting.

\section{P. 19. Mitochondrial metabolism regulates muscle homeostasis in adulthood and ageing Gaia Gherardi, Rosario Rizzuto and Cristina Mammucari \\ aDept. of Biomedical Sciences, University of Padova, Italy}

The second messenger $\mathrm{Ca}_{2+}$ regulates a broad repertoire of cellular processes. Upon physiological stimuli, skeletal muscle mitochondria rapidly and efficiently accumulate $\mathrm{Ca}_{2}+$ into their matrix via an electrogenic pathway, that relies on the driving force of a steep electrochemical gradient. A large [Ca2+]mt peak occurs dynamically in parallel to agonist-induced [ $\mathrm{Ca}_{2}+{ }_{\text {cyt }}$ increases, thanks to the activity of the Mitochondrial Calcium Uniporter (MCU), the highly selective channel responsible for mitochondrial $\mathrm{Ca}_{2}+$ accumulation. MCU positively regulates myofiber size in physiological conditions, and counteracts pathological loss of muscle mass. We have previously demonstrated that skeletal muscle-specific MCU deletion (MCU--) inhibits myofiber mitochondrial $\mathrm{Ca}_{2}+$ uptake, impairs muscle force and exercise performance. Mitochondrial Calcium uptake is required for effective glucose oxidation, as demonstrated by the fact that in muscle-specific MCU-- myofibers 34 oxidative metabolism is impaired and glycolysis rate is increased. The decreased pyruvate dehydrogenase activity is the main trigger of this metabolic rewiring. Although defective, mitochondrial activity is partially sustained by increased fatty acid (FA) oxidation. Here, we have investigated the role of mitochondrial $\mathrm{Ca}_{2+}$ uptake during skeletal muscle aging. We show that mitochondrial $\mathrm{Ca}_{2}+$ accumulation is decreased in 24 months old mice and this condition is accompanied by a decreased pyruvate dehydrogenase activity. We demonstrate a rewiring of skeletal muscle metabolism where mitochondrial activity is sustained by FA oxidation rather than glucose. Further studies are needed to evaluate whether the restoration of glucose as the main fuel for oxidative metabolism will be sufficient to counteract sarcopenia. 
17th IIM Meeting (2019) - Report and Abstracts

Eur J Transl Myol 2020; 30 (4), 9485. doi: 10.4081/ejtm.2020.9485

\author{
P. 20. Characterization of a DUX4 inhibitor in FSHD \\ Paola Ghezzi, Claudia Consonni, Maria Pannese, Valeria Runfola, Davide Gabellini \\ Gene Expression and Muscular Dystrophy Unit, Division of Genetics and Cell Biology, IRCCS San Raffaele Scientific Institute, Milano, \\ Italy
}

Facioscapulohumeral muscular dystrophy (FSHD) is a progressive myopathy due to complex genetic and epigenetic mechanisms. Loss of silencing lead to the ectopic expression of the transcriptional factor double homeobox 4 (DUX4), which is toxic to skeletal muscle causing muscle wasting. DUX4 is a transcription factor normally expressed at the cleavage stage of embryonic development where it activates the expression of genes required for implantation. DUX4 expression is shut off at the 8-cell stage and remains silenced in most somatic tissues. Aberrant gain of DUX4 expression in FSHD muscles induces cell death and muscle wasting. Previous analyses from our group identified the first endogenous protein inhibitor of DUX4 able to bind DUX4 directly, blocking its activity and thus preventing his toxicity in FSHD muscle cells. The aim of my project is to characterize the interaction between DUX4 and MATR3 and identify the minimal MATR3 region necessary and sufficient to inhibit DUX4. I expressed and purified different fragments of DUX4 and the inhibitor in order to test their interaction using vitro pull down experiments. My preliminary results indicate that a 287 aminoacid fragment of the inhibitor is sufficient to interact with the DNA binding domain of DUX4. Smaller fragments are currently being tested to further restrict the minimal regions involved in the interaction. The identified fragments will be verified in cells using co-immunoprecipitation. The ability of the minimal inhibitor fragment to inhibit DUX4 will be tested using apoptosis and myogenic assays in FSHD muscle cells. In parallel, in collaboration with the OSR Biocrystallography group, we will also try to solve the crystal structure of the complex formed between DUX4 and the inhibitor. The final goal of this project is to generate a drug-like molecule based on the identified peptide to test it on pre-clinicals models of the disease as a potential therapeutic strategy for FSHD.

\title{
P. 21. Role of MICAL2 in muscle commitments and rhabdomyosarcoma progression Nefele Giarratana a, Enrico Pozzo a, Stefania Fulle b, Yvan Torrente c and Maurilio Sampaolesi a,d a Translational Cardiomyology Lab, Dept of Dev. and Regeneration, KU Leuven, Belgium - Science, Univ. "G d'Annunzio", Chieti - c Stem Cell Lab, Dept of Pathophysiology and Transplantation, Univ. of Milan - $d$ Human Anatomy Unit, Dept of Public Health, Exp. and Forensic Med., Univ. of Pavia, Italy
}

Rhabdomyosarcoma (RMS) is an aggressive skeletal muscle-lineage tumor, where malignant myoblasts failto exit the cell cycle and are restrained from fusing into syncytial muscle. RMS is also the most common soft tissue sarcoma in pediatric patients and young adults. The treatment is based on multimodal approach of chemotherapy, surgery and/or radiation. However, $30 \%$ of RMS cases are unresponsive to induction chemotherapy and required a combination of more intensive chemotherapy and irradiation. Thus, drug resistance and relapse prevent an effective treatment, and novel strategies targeting key mediators of the molecular machinery involved in the pathogenesis of RMS are necessary. The cytoskeletal actin is responsible for cell morphology and migration, and its organization has been studied in different RMS cell lines. Recently, MICAL2 (microtubule associated monooxygenase, calponin and LIM domain containing 2) a nuclear monooxygenase that promotes depolymerization of F-actin is indicated as a novel human cancer gene controlling mesenchymal to epithelial transition [1]. In addition, MICAL2 is critical for myogenic differentiations since it modifies actin subunits and promotes actin turnover by severing them and preventing repolymerization. Indeed, we demonstrated that modulations of MICAL2 have an impact on muscle filament dynamics and its fine-tuned balance is essential for the regeneration of muscle tissues [2]. Furthermore, we aim to unravel the role of MICAL2 in proliferation, migration and hence the myogenic differentiation incapability in RMS cells. Hence, MICAL2 loss of function studies have been performed on both murine and human RMS cells showing an impact on the RMS proliferation ability. Taken together these data demonstrate that modulations of MICAL2 have an impact both on muscle tissues and on RMS cells, remarking the importance of its balance for muscle regeneration and for cancer progression.

References; [1] Mariotti S, et al. Oncotarget 7: 1808-1825, 2016. [2] Giarratana N, et al. Cell Death and Disease (2020) 11:654 https://doi.org/10.1038/s41419-020-02886-z

\section{P. 22. Exertional Heat Stroke: the role of external Ca2+.}

Barbara Girolami, Laura Pietrangelo, Antonio Michelucci, Matteo Serano, and Feliciano Protasi. 
Exertional/Environmental Heat Stroke (EHS) is a hyperthermic crisis triggered by strenuous physical exercise and/or exposure to environmental heat. These syndromes seem to be caused by an altered intracellular homeostasis of $\mathrm{Ca}_{2}+$ in muscle. Store-Operated $\mathrm{Ca}_{2}+$ Entry (SOCE) is a mechanism that, allowing recovery of extracellular $\mathrm{Ca}_{2}+$ during prolonged activity, influence intracellular $\mathrm{Ca}_{2}+$ levels. We recently demonstrated that exercise leads to formation of Calcium Entry Units (CEUs), intracellular junctions that promote interaction between STIM1 and Orai1, the two proteins that mediate SOCE. Here we tested the hypothesis that exerciseinduced assembly of CEUs may increase the risk of hyperthermic crisis when physical activity is performed in challenging environmental conditions. 4 months old mice were: a) first, divided in 3 experimental groups: control, trained-1m (1 month of voluntary running in wheel cages), and exercised-1h (1 hour of incremental treadmill run); b) second, subjected to an incremental treadmill run of $45 \mathrm{~min}$ at $34^{\circ} \mathrm{C}$ and $40 \%$ humidity. We then: a) measured the internal temperature of mice, which was higher in the pre-exercised groups (trained- $1 \mathrm{~m}$ : $38.9^{\circ} \mathrm{C} 0.33$; exercised-1h: $\left.37.9^{\circ} \mathrm{C} 0.17\right)$ compared to control $\left(38.7^{\circ} \mathrm{C} 0.40\right)$; b) applied an ex-vivo exertional stress protocol to isolated EDL muscles (tetanic stimulation performed at $30^{\circ} \mathrm{C}$ ) and verified that samples from trained-1m and exercised-1h mice generated a tension significantly greater than control; c) Analyzed CEUs by electron microscopy (EM) and verified that EDL muscles of exercised-1h ad trained-1m mice contained a greater number of CEUs. Results collected point to a correlation between the presence of CEUs in muscle fibers and: a) excessive increase in internal temperature during physical exercise performed in challenging conditions; and b) predisposition of muscles to contracture during prolonged stimulation.

\section{P. 23. ACVR2B antagonism as a countermeasure to multi-organ perturbations in metastatic colorectal cancer cachexia \\ Joshua Huot1,2, Fabrizio Pin 2, Ashok Narasimhan 1, Leah Novinger 3, Austin Keith 7, Teresa Zimmers 1-3,5,6, Monte Willis 4-6, Andrea Bonetto 1-3,5,6 \\ 1Department of Surgery, 2 Department of Anatomy, Cell Biology \& Physiology, з Department of Otolaryngology- Head \& Neck Surgery, ${ }_{4}$ Department of Pathology and Laboratory Medicine, 5 Indiana Center for Musculoskeletal Health, 6 Simon Comprehensive Cancer Center, Indiana University School of Medicine, Indianapolis, IN, USA; 7 Zionsville Community High School, Zionsville, IN, USA}

Advanced colorectal cancer (CRC) is often accompanied by the development of liver metastases (LM), as well as cachexia, a multi-organ comorbidity affecting skeletal (SKM) and cardiac muscles, fat, and bone. Activin receptor type 2B (ACVR2B) signaling is known to cause SKM wasting, and its inhibition restores SKM mass and prolongs survival in cancer. Using a recently characterized mouse model, here we tested whether ACVR2B blockade could preserve multiple organs in the presence of metastatic CRC. NSG male mice (8week old) were injected intrasplenically with HCT116 human CRC cells (mHCT116), while sham-operated animals received saline ( $n=5-10 /$ group). Sham a d tumor-bearing mice received weekly injections of ACVR2B/Fc, a synthetic peptide inhibitor of ACVR2B. mHCT116 hosts displayed losses in fat mass $(-79 \%$, $p<0.0001$ ), bone mass $(-39 \%, p<0.05)$ and SKM mass (quadriceps: $-22 \%, p<0.001)$, in line with reduced muscle cross-sectional area (CSA: $-24 \%, p<0.01)$ and plantarflexion force $(-28 \%, p<0.05)$. Further, despite only moderately affected heart size, cardiac function was significantly impaired (ejection fraction (EF)\%: $-16 \%$, $\mathrm{p}<0.0001$; fractional shortening (FS)\%: $-25 \%, \mathrm{p}<0.0001)$ in the mHCT116 hosts. Conversely, ACVR2B/Fc preserved fat mass $(+238 \%, p<0.001)$, bone mass $(+124 \%, p<0.0001)$, SKM mass (quadriceps: $+31 \%$, $\mathrm{p}<0.0001)$ and size (CSA: $+43 \%, p<0.0001)$ and plantarflex on force $(+28 \%, p<0.05)$ in tumor hosts. Cardiac function was also completely preserved in tumor hosts $r$ ceiving ACVR2B/Fc (EF\%: $+19 \%, p<0.0001)$, despite no effect on heart size. RNA-sequencing analysis of heart muscle revealed rescue of genes related to cardiac development and contraction in tumor hosts treated with ACVR2B/Fc. Our metastatic CRC model recapitulates the multi-systemic derangements of cachexia by displaying loss of fat, bone, and SKM along with decreased muscle strength in mHCT116 hosts. Additionally, with evidence of severe cardiac dysfunction, our data support the development of cardiac cachexia in the occurrence of metastatic CRC. Notably, ACVR2B antagonism preserved adipose tissue, bone and SKM, whereas muscle and cardiac functions were completely maintained upon treatment. Altogether, our 36 observations implicate ACVR2B signaling in the development of multi-organ perturbations in metastatic $\mathrm{CRC}$ and further dictate that ACVR2B represents a promising therapeutic target to preserve body composition and functionality in cancer cachexia. 
17th IIM Meeting (2019) - Report and Abstracts

Eur J Transl Myol 2020; 30 (4), 9485. doi: 10.4081/ejtm.2020.9485

\begin{abstract}
P. 24. Role of Menopausal Transition and Physical Activity in Loss of Lean and Muscle Mass: A FollowUp Study in Middle-Aged Finnish Women

Hanna-Kaarina Juppia, Sarianna Sipila a, Neil J. Cronin b,c, Sira Karvinen a, Jari E. Karppinen d, Tuija H.

Tammelin e, Pauliina Aukee f, Vuokko Kovanen a, Urho M. Kujala d and Eija K. Laakkonen a

a Gerontology Research Center and Faculty of Sport and Health Sciences - s Neuromuscular Research Center, Faculty of Sport and Health Sciences, University of Jyväskylä, Finland - c Department for Health, Bath University, UK - $d$ Faculty of Sport and Health Sciences, University of Jyväskylä - e LIKES Research Centre for Physical Activity and Health - $f$ Department of Obstetrics and Gynecology, Pelvic Floor Research and Therapy Unit, Central Finland Central Hospital, Finland
\end{abstract}

During aging muscle mass decreases, which might have an impact on physical function. In women, muscular aging is particularly accelerated in midlife when menopause occurs. During the menopausal transition the concentration of estradiol - the main female sex hormone - decreases, which has been hypothesized to have detrimental effects on skeletal muscle tissue mass and quality. This study investigated the effect of menopausal transition on changes in lean and muscle masses, from the total body to the muscle fiber level, among 47-55-year-old women. Data were used from the Estrogenic Regulation of Muscle Apoptosis (ERMA) study, where 234 women were individually followed from perimenopause to early postmenopause. Hormone levels (estradiol and follicle stimulating hormone), lean body mass (LBM), appendicular lean mass (ALM), single leg lean mass (dual-energy X-ray absorptiometry, DXA), absolute and relative muscle cross-sectional area (CSA) (computed tomography, CT), physical activity level (self-reported and accelerometer-measured) and muscle fiber CSA and fiber type (immunohistochemistry and SDS-PAGE) were assessed at baseline and at early post-menopause. LBM and ALM indexes (LBMI and ALMI) were calculated. Differences in muscle mass variables between peri- and postmenopausal stages were compared with Wilcoxon rank- and paired $t$ test. GEE-modelling was used to study the longitudinal associations of menopausal transition and physical activity on above-mentioned muscle mass variables of interest. Mean follow-up duration was $15.3+/-8.6$ months. On average, participants fulfilled the national recommendations of moderate-to-vigorous physical activity and can therefore be considered active. Significant decreases were seen during the follow-up in LBM $(-0.5 \%)$, LBMI (-0.7\%), ALM (-1.1\%), ALMI (-1.1\%), single leg lean mass $(-1.5 \%)$ and absolute and relative thigh muscle CSA $(-1.0 \%)$ (all $p<0.05)$. No change was observed in the CSA of individual muscle fibers or fiber types. Menopausal status was a significant predictor for all tested muscle mass variables, while physical activity was an additional significant contributor for LBM, ALM, ALMI, single leg lean mass and relative muscle CSA during the follow-up. Menopausal transition was intrinsically associated with loss of muscle mass at multiple anatomical levels, while physical activity was found to be beneficial for the maintenance of skeletal muscle mass during mid-life.

Juppi, H-K, Sipila S, Cronin N J, Karvinen S, Karppinen JE, Tammelin TH, Aukee P, Kovanen V, Kujala UM, Laakkonen E.K. Role of Menopausal Transition and Physical Activity in Loss of Lean and Muscle Mass: A Follow-Up Study in Middle-Aged Finnish Women. J Clin Med 2020, 9, 1588.

\title{
P. 25. Downregulation of dynamin 2 (DNM2) rescues several forms of centronuclear and myotubular myopathies \\ Xenia Massana Munoz a, Vasugi Nattarayan a, Roberto Silva-Rojas a, Belinda Cowling a,b and Jocelyn Laporte a \\ A Institut de Genetique et de Biologie Moleculaire et Cellulaire (IGBMC), INSERM U1258, CNRS UMR7104, Strasbourg University, France - b Dynacure, France
}

Centronuclear and myotubular myopathies (CNM) are severe muscle diseases. The X-linked form, also called myotubular myopathy (XLCNM), is caused by mutations in Myotubularin (MTM1), while two main autosomal forms are due to mutations in either Amphiphysin 2 (BIN1; recessive ARCNM) or Dynamin 2 (DNM2; dominant $A D C N M)$. The underlying pathomechanisms are still unclear and there is no therapy. We hypothesized modulation of a CNM gene may rescue the alterations due to mutations in another CNM gene. Here we show that downregulation of DNM2, via transgenesis, shRNA or antisense oligonucleotides (ASO), efficiently rescues the motor defects of mouse models of these different CNM forms, including the Mtm1 knock-out, the Bin1 muscle-specific knock-out, and two Dnm2 knock-in of a mild (R465W) or severe (S619L) DNM2 mutations. Moreover, Dnm2 targeting also rescues the typical CNM histopathology displayed by the disease models, including myofiber hypotrophy and organelles mis-positioning. These results indicate that MTM1, BIN1 and DNM2 regulate muscle organization, maintenance and function through a common pathway, and support that 
17th IIM Meeting (2019) - Report and Abstracts

Eur J Transl Myol 2020; 30 (4), 9485. doi: 10.4081/ejtm.2020.9485

overactivation of DNM2 is at the basis of the different CNM forms. DNM2 downregulation thus represents a common therapy to improve at least 4 different forms of centronuclear myopathies.

\section{P. 26. Amphiphysin 2 (BIN1) modulates dynamin 2 in vivo and rescues dynamin 2-centronuclear myopathy \\ Valentina Maria Lionello a, Christine Kretz a, Evelina Edelweiss a, Corinne Crucifix a, Nadia Messaddeq a, Suzie Buono a, Pascale Koebel a, Belinda S. Cowling a, Marc Bitoun b, Jocelyn Laporte a \\ a Institut de Genetique et de Biologie Moleculaire et Cellulaire (IGBMC), INSERM U1258, CRNS UMR7104, Strasbourg University, Illkirch, France - b Institute of Myology, Research Center for Myology, INSERM, UMRS974, Sorbonne Universite, Paris, France.}

The mechanoenzyme dynamin 2 (DNM2) is a ubiquitously expressed GTPase crucial for intracellular organization and membrane trafficking. Dominant mutations in DNM2 lead to the autosomal dominant form of centronuclear myopathy (DNM2-CNM), a severe incurable muscle disease characterized by defects in organelle positioning in myofibers. Currently, little is known on the in vivo functions of DNM2 in muscle and there is no therapy available for DNM2-CNM patients. Here we modulate human amphiphysin 2 (BIN1), a membrane remodeling protein mutated in other forms of CNM, in DNM2-CNM mild (Dnm2RW/+) and severe (Dnm2RW/RW) murine models through transgenesis or adenoassociated virus (AAV)-mediated transduction. The overexpression of BIN1 improved muscle atrophy, and the main histopathological and ultrastructural features of $D n m 2 \mathrm{RW} /+$ mice. Moreover, increasing human BIN1 level rescued the perinatal lethality and survival of Dnm2RW/Rw mice. In vitro experiments showed that BIN1 is necessary for DNM2 recruitment to membrane tubules, and that BIN1 together with DNM2 modulates tubule fission. In conclusion, BIN1 and DNM2 are part of a common pathway needed to maintain skeletal muscle membrane homeostasis. With this study, we identify BIN1 as a novel therapeutical target for autosomal dominant centronuclear myopathy patients.

\section{P.27. Dual role of eEF2k/eEF2 pathway in development of disuse-induced atrophy in skeletal muscle. Yulia Lomonosova a,b, Natalia Vilchinskaya c, Catheryn Wooi Fang Lim a,b, Svetlana Belova c, Thomas \\ Roberts a,b, Matthew Wood a,b. \\ a Department of Paediatrics, University of Oxford, United Kingdom. sMDUK Oxford Neuromuscular Centre, United Kingdom. c Institute of Biomedical Problems, Russian Academy of Sciences, Russia.}

Prolonged periods of skeletal muscle inactivity during space flight, traumatic injuries, extended bed rest, diseases including AIDS, cancers, neurological disorders and age-related muscle weakness (sarcopenia) leads to muscle atrophy [Fanzani et al., 2012]. It is believed that impairment in the regulation of skeletal muscle protein synthesis is primary cause of disuse muscle atrophy in healthy men [Phillips et al., 2009, Wall et al., 2013]. The principal mechanism underlying the reduction of protein synthesis rate in atrophied skeletal muscle is largely unknown. Here, we focus on the role of translation elongation factor 2 (eEF2) and inhibitory transphosphorylation at T56 by its specific kinase (eEF2k) in the protein synthesis impairment. Different periods of atrophy development in m.soleus have been investigated utilizing rat hindlimb suspension (HS) model. We demonstrated two different stages of the eEF2k/eEF2 cascade misregulation during muscle wasting, observing increase in eEF2k expression and inhibition of eEF2. We showed that eEF2k activation upon disuse is a Ca2+- dependent process with involvement of Cav1 L-type calcium channels, utilising BAPTAAM and nifedipine. We applied A484954 and $\mathrm{NH} 125$ small molecules to manipulate with eEF2k/eEF2 pathway activity, retaining the protein synthesis rate in the disused muscle. We demonstrated that overexpression of eEF2k in myoblasts resulted in suppression of ribosomal protein S6 kinase (p70S6k) activity towards its downstream targets, such as ribosomal protein S6 (rpS6) and eukaryotic translation initiation factor 4B (elF4B), leading to the reduction in global protein synthesis. We showed that both phosphorylated and total eEF2 levels are reduced in a glucocorticoid-induced atrophy model in myotubes. Lastly, given that alterations in eEF2 activity might influence on accumulation of molecular markers of disuse atrophy such as $\mu$-calpain, and E3ubiquitin ligases, muscle atrophy F-box protein (MAFbx/atrogin-1) and muscle RING Finger-1 (MuRF-1) [Tischler et al., 1990; Bodine et al., 2001; Dupont-Versteegden et al., 2006; Enns et al., 2007], we were motivated to analyse protein expression of the proteases. We observed that $\mu$-calpain and the E3-ubiquitin ligases responded differently to changes in eEF2k-eEF2 cascade activity during HS. Notably, enhanced inhibition of eEF2 upon HS resulted in suppression of both MAFbx/atrogin-1 and MuRF-1. The present study provides a new understanding of the mechanisms responsible for decreased protein synthesis during skeletal 
muscle atrophy, specifically, the contribution of increased expression and activity of eEF2k to the impairment of p70S6k function and reduced translation elongation. Furthermore, our data suggest that inhibition of eEF2 can decrease protein expression of individual proteins, for example, E3-ligases MAFbx and MuRF-1, which should be considered in the development of therapies to prevent muscle loss during wasting.

Funding Statement: this study was supported by Russian Foundation for Basic Research and Medical Research Council.

\section{P. 28. Polyglutamine-expanded androgen receptor alters excitation-contraction coupling machinery and calcium dynamics in skeletal muscle \\ Caterina Marchioretti 1,2, Mathilde Chivet 3, Marco Pirazzini 1, Giulia Zanetti 1, Gaia Gherardi 1, Leonardo \\ Nogara 2, Gaia Butera 1, Cristina Mammucari 1, Anna Raffaello 1, Bert Blaauw 2, Gianni Soraru 4, Maria Pennuto $1,2,4$. \\ 1. Dept. of Biomedical Sciences (DBS), University of Padova - 2. Veneto Institute of Molecular Medicine (VIMM) - 3. Dulbecco Telethon Institute, Centre for Integrative Biology (CIBIO), University of Trento - 4. Dept. of Neuroscience (DNS), University of Padova, Italy}

Spinal and bulbar muscular atrophy (SBMA) is a X-linked disease caused by polyglutamine (polyQ) expansions in the androgen receptor (AR) gene. For a long time, SBMA has been thought to be primary a motoneuron disorder but in the last decade several evidences have demonstrated that skeletal muscle plays a key role in the development of this disease. Although clinical and experimental evidence highlight a primary role for skeletal muscle in the onset and progression of disease, the molecular mechanisms underlying SBMA muscle atrophy are still unclear. Moreover, skeletal muscle is easier to treat respect to the motor neuron, suggesting that therapies targeting this tissue can have a significant impact on patients suffering from this disease. We show that polyQ-expanded AR alters intrinsic muscle force generation before denervation. Reduced muscle force was associated with a switch in fiber-type composition, disrupted muscle striation, altered calcium (Ca++) dynamics in response to muscle contraction, and aberrant expression of excitationcontraction coupling (ECC) machinery genes in transgenic, knock-in and inducible SBMA mice and patients. Acute suppression of AR activation by surgical castration elicited similar ECC gene expression changes in normal mice, suggesting that AR regulates the expression of these genes in physiological conditions. Importantly, treatment to suppress polyQ-expanded AR expression restored ECC gene expression back to normal. Bioinformatic analysis revealed the presence of androgen-responsive elements on several genes involved in muscle function and homeostasis. Experimental evidence showed AR dependent regulation of expression and promoter occupancy of the most up-regulated gene from transcriptomic analysis in SBMA muscle. These observations reveal an unpredicted role for $\mathrm{AR}$ in the regulation of expression of genes involved in muscle contraction and Ca++ dynamics, a level of muscle function regulation that is disrupted in SBMA muscle, yet restored by pharmacologic treatment.

\section{P. 29. Allele specific silencing by RNAi of R92Q and R173W mutations in cardiac troponin $T$ Loredana Migliore a, Federico Galvagni b, Enrico Pierantozzi a, Daniela Rossi a and Vincenzo Sorrentino a a Department of Molecular and Developmental Medicine - s Department of Biotechnology, Chemistry and Pharmacy, University of Siena, Italy}

Many familial forms of hypertrophic and dilated cardiomyopathy (HCM and DCM) are linked to autosomal dominant mutations in genes encoding sarcomeric proteins. Several efforts have been done to develop therapeutic approaches for these patients, including the design of Allele-Specific Silencing approaches by RNA interference (ASP-RNAi). ASP-RNAi represents a powerful and promising strategy to counteract genetic defects by using duplex small interfering RNAs (siRNAs) to target mutant alleles with minimal suppression of the corresponding wild-type allele. Concerning mutations in sarcomeric proteins associated to HCM and DCM, we focused on the R92Q and R173W missense mutations in the cardiac Troponin $\mathrm{T}$ gene (cTnT). We developed an ASP-RNAi strategy to specifically knock down mutant alleles carrying the R92Q and the R173W missense mutations. Following siRNA design and generation, the specific silencing of the mutant allele was tested by a luciferase reporter gene assay and further confirmed on a HEK293T cell model expressing either the wild type or mutant cTnT alleles. Sets of siRNA fully complementary to the target sequence or siRNA containing single-base mismatches downstream the targeted mutations were analyzed and evaluated. Results obtained showed that optimal allele discrimination was obtained with the use of siRNA containing a singlebase mismatch both for the R92Q and the R173W mutant alleles. Following optimal standardization of this technique, we are planning to test these siRNAs in patient specific iPSC-derived cardiomyocytes heterozygous 
for R92Q or the R173W missense mutations to evaluate the ability of allele silencing to rescue functional parameters in these cells.

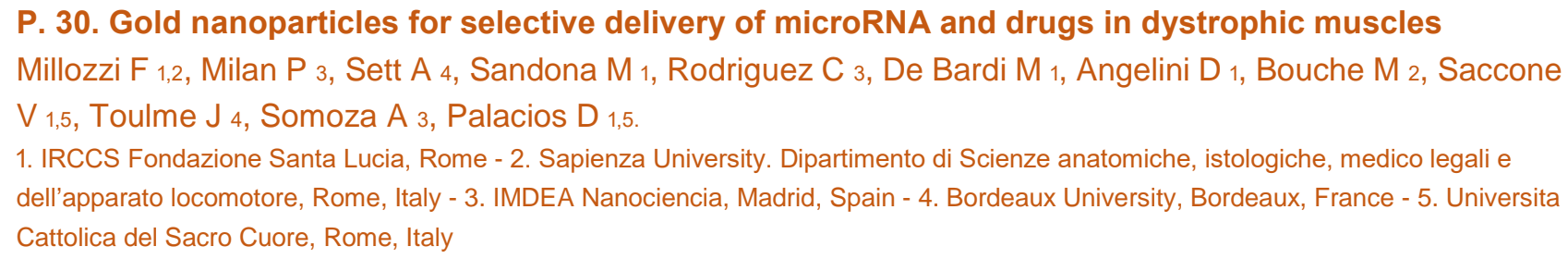

Duchenne muscular dystrophy (DMD) is a muscle disorder caused by mutations in dystrophin gene, an important component of the sarcoglycan complex that protects muscle fibers from damage. At early stages of the disease, muscles are characterized by several cycles of degeneration/regeneration while at late stages the muscle regeneration potential is exhausted and muscles are replaced by collagen deposition and fat infiltration. Currently, there is no cure available and therapeutic strategies focused on improving muscle regeneration through muscle stem cells (satellite cells) activation, and on reducing fibrotic deposition are under investigation. Here we propose a new therapeutic approach based on the potential of novel nanosystems as efficient biocarriers to increase and/or extend the regenerative potential of skeletal muscle. In particular, we will use functionalized gold spherical nanoparticles (AuNPs) to delivery microRNAs and drugs into muscle stem cells. Further, we will conjugate our nanosystems to an aptamer selected against alpha7/beta1 integrin dimers, a satellite cell biomarker. The overall goal of this technology is to deliver therapeutic agents to specific target sites in vivo in a controlled manner. Preliminary ex vivo experiments using $\mathrm{C} 2 \mathrm{C} 12$ cells and freshly isolated satellite cells indicate that aptamer-conjugated AuNPs containing microRNAs are functional and able to modulate satellite cells function. In addition, biodistribution experiments in dystrophic (mdx) mice showed that the developed nanotechnology is able to efficiently target satellite cells upon systemic delivery in vivo. Finally, delivery of AuNPs conjugated with miR206, a key miRNA involved in regulating satellite cells function, increases muscle regeneration and reduces fibrosis in $\mathrm{mdx}$ mice. In conclusion, we have developed a novel delivery system able to release oligonucleotides and/or drugs specifically in satellite cells. Our data indicate that aptamer conjugated, microRNA-containing nanoparticles may represent a new therapeutic tool to improve regeneration of dystrophic muscles.

\section{P. 31. Characterization of the transcription factor Nfix regulatory mechanisms in skeletal myoblasts Giada Mura, Michela Lapi, Marielle Saclier, Chiara Bonfanti, Marco Nardini and Graziella Messina Department of Biosciences, University of Milan, Italy}

Nfix is a transcription factor that drives the transition from embryonic to fetal myogenesis in myoblasts. In the adult, Nfix plays a role in the maintenance of the correct timing of skeletal muscle regeneration upon injury, controlling both satellite cells' differentiation and the skewing from M1 to M2 macrophages. Consequently, silencing Nfix in dystrophic mice leads to a morphological and functional amelioration of the dystrophic phenotype by slowing down the degeneration-regeneration cycles and switching muscle fibers towards a slow twitching phenotype. In this work, we demonstrate that Nfix is regulated by post-translational modifications. In particular, we proved the existence of a phosphorylated form of Nfix in myoblasts. This modification appears concomitantly with the expression of Nfix at $14.5 \mathrm{dpc}$ in mouse fetal myoblasts when Nfix guides the secondary myogenesis. This may indicate that Nfix must be phosphorylated to explicate its role in myoblasts. Several proteomics data from different cell types indicate the presence of multiple phosphorylation sites on Nfix protein sequence. The evident molecular weight shift observed for phospho-Nfix suggests that the protein may bear more than one phosphorylated amino acid. To evaluate this possibility and to disclose the functional meaning of these phosphorylations we are now performing mutagenesis analyses on these sites. Unexpectedly, the levels of the phosphorylated Nfix band specifically and significantly drop in myoblasts upon a 4 hours exposure to proteasome inhibitors, followed by Nfix mRNA reduction at later time points. We propose that proteasome inhibition stabilizes a phosphatase responsible for the removal of such modification and that in the absence of phosphorylation Nfix transcription is eventually destabilized. Importantly, ERK1/2 phosphorylation significantly drops after $4 \mathrm{~h}$ of bortezomib treatment, a proteasome inhibitor, in $\mathrm{C} 2 \mathrm{C} 12$. We already demonstrated that pERK is involved in the regulation of Nfix during development, and this data could lead to a better understanding of the molecular mechanisms behind pERK-Nfix interplay. Know your enemy: the focus and importance of this 
study rely on the clarification of Nfix physiological regulatory mechanisms, which will allow to manipulate its transcriptional activity and/or DNA-binding capacity, for future pharmacological studies aiming at its inhibition in a dystrophic context.

P. 32. Identification and inhibition of a novel chromatin remodeling protein blocks DUX4 expression and rescues myogenic defects in FSHD muscular dystrophy

Emanuele Mocciaro, Roberto Giambruno, Stefano Micheloni, Cristina Consonni, Maria Pannese, Valeria

Runfola, Giulia Ferri, Davide Gabellini

Gene Expression and Muscular Dystrophy Unit, Division of Genetics and Cell Biology, IRCCS San Raffaele Scientific Institute, Milano, Italy

Facioscapulohumeral muscular dystrophy (FSHD) is the most prevalent progressive myopathy that afflicts both children and adults regardless of the gender. FSHD is caused by aberrant gain of expression of the double homeobox 4 (DUX4) gene. DUX4 misexpression triggers the activation of a pro-apoptotic transcriptional program leading to muscle wasting. As today, no cure or therapeutic option are available to FSHD patients. Our laboratory previously showed that the long non-coding RNA DBE-T is required for aberrant DUX4 expression in FSHD. Using affinity purification followed by proteomics, we identified a chromatin remodeling protein as a novel DBE-T interactor and a major player required for the biological activity of the IncRNA. Through this work, we demonstrated that the novel DBE-T binding protein is required for DUX4 activation in FSHD muscle cells. Moreover, targeting the novel DBE-T binding protein rescues cell viability and myogenic differentiation of FSHD muscle cells. Remarkably, we obtained similar result by pharmacological inhibition of the novel DBE-T binding protein. These results not only support a pivotal role of the DBE-T binding protein but also identified a novel and druggable DUX4 activator as an innovative therapeutic approach for FSHD.

P. 33. Quantitative proteomics to unravel disease mechanisms and adaptive responses in M E L A S and M E R R F

Marta Murgia ab, Jing Tan c, and Thomas Klopstock cd

a Department of Biomedical Sciences, University of Padova, Italy -

Institute of Biochemistry, Martinsried - c Friedrich Baur Institute, Department of Neurology, University of Munichy - a German Center for

Neurodegenerative Diseases (DZNE), Munich, Germany

Numerous pathological mutations in mitochondrial tRNA genes are associated with a wide panoply of diseases. It is unclear, however, why different tRNA point mutations lead to very different disease phenotypes. We here apply mass spectrometry-based proteomics to the direct comparison of muscle biopsies from patients diagnosed with MELAS (Mitochondrial Encephalomyopathy, Lactic Acidosis, and Stroke-like episodes) and MERRF (Myoclonus Epilepsy with Ragged-Red Fibers). These disorders are caused by a mitochondrial DNA point mutation in the Leu $(3243 A>G)$ and Lys $(8344 A>G)$ mitochondrial tRNA respectively. We aim at providing a system view of the MELAS and MERRF muscle proteome, highlighting differences that may underlie the profoundly different clinical manifestation of these mitochondrial disorders.

\section{P. 34. Trabectedin and lurbinectedin extend survival of mice bearing C26 colon adenocarcinoma,} without affecting tumor growth or cachexia

Giorgio Aquila a, ${ }^{*}$, Andrea David Re Cecconi a, ${ }^{*}$, Mara Forti a, Roberta Frapoll ib, Ezia Bello b, Deborah Novelli c, Ilaria Russo c, Simonetta Andrea Licandro b, Lidia Staszewsky c, Giulia Benedetta Martinelli a, Laura Talamini d, Laura Pasetto d, Andrea Resovi e, Raffaella Giavazzi b, Eugenio Scanziani f,g, Giorgia Careccia h, Emilie Venereau h, Serge Masson c, Roberto Latini c, Maurizio D'Incalc ib and Rosanna Piccirillo a, ${ }^{\star \star}$

a Department of Neurosciences, s Department of Oncology, c Department of Cardiovascular Medicine, a Department of Biochemistry and Molecular Pharmacology, Mario Negri Institute for Pharmacological Research IRCCS, Milan, e Mario Negri Institute for Pharmacological Research IRCCS/Department of Oncology, Bergamo, Italy f Dipartimento di Medicina Veterinaria, Università di Milano, g Mouse and Animal Pathology Lab (MAPLab), Fondazione UniMi, Università di Milano, nDivision of Genetics and Cell Biology, IRCCS San Raffaele Scientific Institute, Milan, Italy * These authors contributed equally to this work. ** corresponding author. 
Trabectedin (ET743) and lurbinectedin (PM01183) limit the production of inflammatory cytokines that are elevated during cancer cachexia. Mice carrying C26 colon adenocarcinoma display cachexia (i.e., premature death and body wasting with muscle, fat and cardiac tissue depletion), high levels of inflammatory cytokines and subsequent splenomegaly. We tested whether such drugs protected these mice from cachexia. Ten weekold mice were inoculated with C26 cells and three days later randomized to receive intravenously vehicle or $0.05 \mathrm{mg} / \mathrm{kg}$ ET743 or $0.07 \mathrm{mg} / \mathrm{kg}$ PM01183, three times a week for three weeks. ET743 or PM01183 extended the lifespan of C26-mice by $30 \%$ or $85 \%$, respectively, without affecting tumor growth or food intake. Within 13 days from C26 implant, both drugs did not protect fat, muscle and heart from cachexia. Since PM01183 extended the animal survival more than ET743, we analyzed PM01183 further. In tibialis anterior of C26-mice, but not in atrophying myotubes, PM01183 restrained the NF-KB/PAX7/myogenin axis, possibly reducing the pro-inflammatory milieu, and failed to limit the C/EBPB/atrogin-1 axis. Inflammation-mediated splenomegaly of C26-mice was inhibited by PM01183 for as long as the treatment lasted, without reducing IL-6, M-CSF or IL-1B in plasma. ET743 and PM01183 extend the survival of C26-bearing mice unchanging tumor growth or cachexia but possibly restrain muscle-related inflammation and C26-induced splenomegaly.

\section{P. 35. The discovery of Calcium Entry Units (CEUs). \\ Laura Pietrangelo, Simona Boncompagni, and Feliciano Protasi \\ CAST, Center for Advanced Studies and Technology, University G. d'Annunzio of Chieti-Pescara, Chieti, Italy.}

Store-operated $\mathrm{Ca}_{2}+$ entry (SOCE) is a mechanism, first described in non-excitable cells, triggered by depletion of intracellular $\mathrm{Ca}_{2+}$ stores (endoplasmic reticulum, ER). The two essential molecular players in SOCE are STIM1, the $\mathrm{Ca}_{2+}$ sensor in the ER, and Orai1, a $\mathrm{Ca}_{2+}$ permeable channel of external membranes. SOCE is also well-documented in skeletal muscle, where it is also mediated by STIM1 and Orai1. However,the subcellular sites of STIM1-Orai1 interaction in skeletal muscle fibers were not specifically investigated for more than a decade after SOCE was first detected in 2001. Searching for SOCE sites, we discovered new intracellular junctions (i.e. not previously identified) between sarcoplasmic reticulum (SR) and transverse-tubules (TTs), which contains STIM1 and Orai1 and that assemble during exercise and disassemble following recovery. Presence of these new junctions correlates with a) increased fatigue resistance of muscles during highfrequency stimulation in presence of external $\mathrm{Ca}_{2}+$ and b) augmented rate of $\mathrm{Mn}_{2+}$ quench of Fura-2 fluorescence, the gold standard technique used to measure SOCE. We also discovered that CEUs are constitutively assembled in muscle fibers of calsequetrin-1 knockout (CASQ1-null) mice, which are prone to fast depletion when repetitively stimulated. Our work represents a pioneer study that identified new dynamic intracellular junctions that works as $\mathrm{Ca}_{2}+$ Entry Units (CEUs). Assembly of CEUs formation is driven either by prolonged exercise (a mechanism possibly important for the delay of muscle fatigue) and/or by depletion of intracellular stores (which may occur during fatigue or also a result of genetic modifications; ex.: CASQ1 ablation). As altered SOCE and mutations in STIM1 and Orai1contributes to muscle dysfunction in ageing and various myopathies (including tubular aggregate myopathy, TAM), our findings may also have implications for the understanding of mechanisms causing improper muscle function.

\section{P. 36. Mitochondrial calcium signaling in Duchenne muscular dystrophy \\ Federica Placa, Gaia Gherardi, Rosario Rizzuto, Cristina Mammucari \\ aDepartment of Biomedical Sciences, University of Padua, Italy}

Duchenne muscular dystrophy (DMD) is a progressive and X-linked muscle wasting disease caused by the lack of dystrophin, essential protein for sarcolemma stability. Calcium dysregulation, a central event in dystrophic muscles, contributes to muscle degeneration, altered regeneration and mitochondrial dysfunction. Indeed, high cytosolic calcium levels trigger mitochondrial calcium overload, which determines the opening of the mitochondrial permeability transition pore and eventually cell death. Mitochondrial calcium uptake is controlled by a multiprotein complex i.e. the Mitochondrial Calcium Uniporter (MCU). Previous studies demonstrated that in mdx mice, a widely used animal model of DMD, cytosolic calcium transients and mitochondrial calcium uptake are increased compare to wild type mice. MCU silencing reduced central nucleated regenerating fibers in tibialis anterior (TA) muscles of mdx mice. Moreover, fiber size analysis of TA muscles indicated that the myofiber area was increased upon MCU silencing. Finally, a fiber size distribution analysis revealed decreased heterogeneity in treated muscles compare to controls. In conclusion, our data 
17th IIM Meeting (2019) - Report and Abstracts

Eur J Transl Myol 2020; 30 (4), 9485. doi: 10.4081/ejtm.2020.9485

support the hypothesis that a reduction of mitochondrial calcium uptake could restore mitochondrial function and quality in DMD muscles.

\section{P. 37. RANKL blockade reduces cachexia and bone loss induced by non-metastatic cancer \\ Fabrizio Pin 1, Alexander J. Jones 2, Joshua R. Huot 3, Ashok Narasimhan 3, Teresa A. Zimmers 1,2,3,4,5, Lynda F. Bonewald 1,4,5, Andrea Bonetto 1,2,3,4,5 \\ 1Department of Anatomy, Cell Biology and Physiology; 2 Department of Otolaryngology-Head \& Neck Surgery; 3 Department of Surgery; ${ }_{4}$ Simon Comprehensive Cancer Center; 5 Indiana Center for Musculoskeletal Health, Indiana University School of Medicine, Indianapolis, IN, USA.}

Tumor- and bone-derived soluble factors have been proposed to participate in the disregulation of skeletal muscle size and function in cachexia. We previously showed that mice bearing ovarian cancer (OvCa) exhibit cachexia associated with marked bone loss, whereas bone-targeting agents, such as bisphosphonates, are able to preserve muscle mass in animals exposed to anticancer drugs. To the extent of isolating new regulators of bone and muscle in cachexia, here we demonstrated that subjects affected with OvCa display evidence of cachexia and increased bone turnover (CTX-I) in line with elevated levels of receptor activator of NFKB ligand (RANKL), a well-known regulator of osteoclast-dependent bone resorption. Similarly, mice carrying OvCa presented high RANKL in both blood and ascites. We found that RANKL is sufficient to cause myotubes atrophy, along with modulation of inflammatory signaling pathways, and elevated RANKL is sufficient to induce muscle atrophy, muscle weakness and bone loss in healthy mice. Additionally, mice implanted with RANKLoverexpressing C26 colorectal cancer cells displayed exacerbated muscle wasting and bone loss compared to animals carrying wild-type C26 tumors. Anti-RANKL neutralizing antibodies counteracted myotube atrophy induced by ES-2 OvCa cells, as well as improved muscle mass and strength, preserved bone mass and normalized RANKL/OPG expression in OvCa hosts. Finally, bone preservation by the bisphosphonate zoledronate improved muscle mass and strength in OvCa hosts, in association with correction of the RANKL/OPG ratio in bone. Our data contributes to identify RANKL as a new player in driving the musculoskeletal alterations that characterize cancer cachexia, as well as a novel therapeutic target for the treatment of the muscle and bone complications associated with RANKL-expressing non-metastatic cancers. Furthermore, our results showing beneficial muscle and bone effects resulting from administration of zoledronate and anti-RANKL antibodies opens up new opportunities for the testing of safe, well-characterize and FDA-approved anti-resorptive drugs as standard of care in the treatment of muscle and bone defects in OvCa.

P. 38. Unraveling the use of anti-tumorigenic pro-differentiating microRNAs in pediatric rhabdomyosarcoma

Enrico Pozzo a, Merve Elmastas a, Gabriele Sassi a, Margot Claes a, Nefele Giarratana a, Jason Yustein b, Anne Uyttebroeck c and Maurilio Sampaolesi a,d

a Department of Development and Regeneration, KU Leuven, Belgium - s Department of Pediatrics, Baylor College of Medicine, Houston,

TX, USA - c Department of Pediatric Oncology, UZ Leuven, Belgium - a Department of Public Health, Experimental and Forensic Medicine, University of Pavia, Italy

Rhabdomyosarcoma (RMS) is the most common soft tissue sarcoma of childhood arising from undifferentiated skeletal muscle cells from uncertain origin. Currently used therapies are poorly tumor-specific and fail to tackle the molecular machinery underlying the tumorigenicity and uncontrolled proliferation of RMS1. We recently found that pleiotropic oncosuppressor miRNAs have a positive impact to reduce RMS proliferation and invasiveness. Here, we identified a novel combination of oncosuppressor miRNAs poorly present in RMS patients. We then assessed the effects of this miRNA combination on stemness, cell cycle, migration, metabolism and differentiation in both mouse and human RMS. We then sequenced miRNA-treated mouse and human RMS cell lines to identify the up- and downregulation mediated by the novel therapeutic miRNA combinations. Subsequently, we addressed the effects of miRNAs in vivo by performing syngeneic transplant of the pre-treated RMS cell line in C57BI/6. In conclusion, in our study we identified a novel miRNA combination tackling the tumorigenic features of RMS by reducing its cancer stemness, proliferation and metastatic potential. Moreover, we observed an increased expression of genes related to cilium formation2,3, which hints at an increased differentiated phenotype of miRNA-treated RMS. Finally, we identified novel antitumorigenic 
17th IIM Meeting (2019) - Report and Abstracts

Eur J Transl Myol 2020; 30 (4), 9485. doi: 10.4081/ejtm.2020.9485

therapeutic targets that can be exploited by means of molecular targeted therapies for future clinical applications.

References: [1] Yohe ME, Heske CM et al. Pediatr Blood Cancer. 2019 Oct;66(10):e27869; [2] Zingg D et al. Cancer Cell. 2018 Jul 9;34(1):69-84.e14; [3] Fu W et al. Proc Natl Acad Sci U S A. 2014 Jun 24;111(25):9151-6.

\author{
P.39. Investigation of the different outcomes of vitamin D supplementation to counteract skeletal \\ muscle loss in sarcopenia and cachexia \\ Tommaso Raiteri 1, Maraiza Alves Teixeira 1,2, Marilisa De Feudis 1, Andrea Scircoli 1, Laura Salvadori 1,2, \\ Flavia \\ Prodam 3, Simone Reano 1,2, Nicoletta Filigheddu 1,2. \\ ${ }_{1}$ Department of Translational Medicine, University of Piemonte Orientale; 2 Istituto Interuniversitario di Miologia (IIM); 3 Department of \\ Health Sciences, University of Piemonte Orientale, Novara, Italy
}

Skeletal muscle wasting represents one of the main overlapping features between the physiological agerelated sarcopenia and cachexia, which often associates with an underlying disease, such as cancer. Hormonal network dysregulation co-occurs in both sarcopenia and cachexia and could participate in skeletal muscle decline. In humans, vitamin $\mathrm{D}(\mathrm{VD})$ is one of these hormones often dysregulated and reduced and, given the relationship between VD levels and muscle mass and functionality, its supplementation has been proposed as a therapeutic strategy to prevent or treat muscle wasting in both sarcopenia and cachexia. Notably, in elderly subjects, VD supplementation is able to restore muscle strength and prevent muscle mass loss 1 . On the contrary, VD administration is ineffective to counteract cancer cachexia associated-muscle wasting both in patients and in animal model s2,3. We recently demonstrated in vitro that different vitamin $\mathrm{D}$ metabolites have opposite effects on $\mathrm{C} 2 \mathrm{C} 12$ myotubes, depending on the sites of hydroxylation or doses. In detail, 25-hydroxy VD has protective activity and 1,25-dihydroxy VD is atrophic, likely because of a differential modulation of 24hydroxylase, and 24,25- dihydroxy VD itself could have divergent effects, either atrophic or hypertrophic, depending on its concentration 4 . We therefore hypothesize that a dysregulated metabolism of VD in cachectic subjects, leading to different production of protective versus pro-atrophic metabolites could explain the lack of effectiveness of VD supplementation in cachexia. The investigation of the putative different molecular mechanisms involved in VD elicited effects in skeletal muscle in sarcopenia versus cachexia could help to understand the lack of efficacy of VD supplementation in cachexia and, hopefully, to tailor patient-specific therapeutic interventions.

[This study was funded by Research program "Departments of Excellence 2018-2022", AGING Project - Department of Translational Medicine, Universita del Piemonte Orientale, Italy.]

[1] Iolascon et al., Adv Ther. 2017;34:744-752; [2] Scher HI et al., J Clin Oncol. 2011;29:2191-2198; [3] Camperi A et al., Oncotarget. 2017;8:21778-21793. [4] Sustova H et al., Acta Physiol. 2019; 226: 1-10.

43

\title{
P. 40. Macrophages expressing Nfix promote the progression of Muscular Dystrophy Marielle Sacliea, Giulia Temponi, Elena Bertolloti, Chiara Bonfanti,and Graziella Messina Department of Biosciences, University of Milan, Italy
}

Skeletal muscle regeneration requires specific interactions between macrophages (MPs) and myogenic cells at precise time windows. Nfix is a transcription factor expressed by several cell types and it has been shown as necessary to muscle regeneration in total Nfix KO mice. We showed that Nfix expression MPs is required for successful muscle regeneration after acute injury du to a defect of acquisition of anti-inflammatory phenotype and correct function through myogenic cells. More precisely, we demonstrated Nfix is expressed by MPs upon RhoA-ROCK1 dependant-phagocytosis of apoptotic myogenic cells. Thus, we identified Nfix as a key-link between the phagocytic process and the pro- to anti-inflammatory switch, necessary for the resolution of inflammation. In the laboratory, we previsouly demonstrated that the silencing Nfix in dystrophic mice rescues muscular dystrophy by delaying muscle regeneration. Interestingly, we observed an increase of the number of MPs expressing Nfix in both $\mathrm{mdx}$ and sgca $(--)$ dystrophic mice during the progression of the pathology. As MPs are involved in the worsening of muscular dystrophies, we decided to generate a dystrophic mice deleted for Nfix only in MPs, the LysMcre: Nfixf//fl :sgca(-I-) mice. We observed an amelioration of the dystrophic phenotype in terms of muscle structure (myofiber caliber, centro-nucleation) but also a decrease of 
fibrotic deposition (collagen I) that persist over 6 months of life. More importantly, while dystrophic mice are characterised by a heterogenous cycles of necrosis-regeneration, we observed that the LysMcre :Nfixfl/fl :Sgca(I-) mice always exhibit a low number of necrotic myofiber. Moreover, we observed a decrease of in the number of FAPs and an increase in the number MPs expressing TNFa. Interestingly, the pharmaceutic activation of the RhoAROCK1 pathway in sgca(--) dystrophic mice induces the increase of MPs expressing Nfix that leads to the worsening of the dystrophy, while the intra-muscular injection of LysMcre:Nfixfl/f MPs seems to delay the progression of the disease. With this study, we showed that playing on MPs is sufficient to observe muscle improvement in a dystrophic context, by playing both on myogenic cells and FAPs, and place Nfix as an important player of MPs and Satellite Cells function in dystrophies.

\section{P. 41. Natural products to counteract muscle atrophy}

Laura Salvadori a,b, Manuela Mandrone c, Tommaso Manenti d, Catia Ercolani d, Luca Cornioli d, Mariacaterina Lianza c, Paola Tomasi c, Simone Rean Oa,b, Sara Chiappalupi b,e, Ferruccio Poli c, Nicoletta Filigheddu a,b, Guglielmo Sorci b,e and Francesca Riuzzi b,e

A Dept. Translational Medicine, University of Piemonte Orientale, Novara; s Interuniversity Institute of Myology (IIM), Perugia; c Dept. Pharmacy and Biotechnology (FaBiT), University of Bologna; a Biokyma Laboratory srl, Anghiari ; e Dept. Experimental Medicine, University of Perugia, Italy

The loss of skeletal muscle mass and strength, defined as muscle atrophy, represents an unresolved enormous medical problem associated with diffuse pathological conditions and aging (sarcopenia) [1,2]. The decrease of bone mass (osteoporosis) is also strictly connected to muscle functionality during aging [3]. The unbalance between myofibrillary protein breakdown (especially myosin heavy chain, MyHC) and synthesis, and the reduction of regenerative potential are the main causes of muscle wasting [1,2]. In the last decades, an increasing interest has been devoted to identify natural active metabolites with proved pharmacological activities, useful in maintenance of healthy longevity and in the prevention/treatment of various diseases [4]. We identified an herbal formulation composed by hydroalcoholic extracts from W. somnifera (W), S. marianum (S) and $T$. foenum-graecum ( $\mathrm{T}$ ) with remarkable ability to prevent the reduction of myotube size in well characterized in vitro experimental models mimicking muscle atrophy induced by pro-inflammatory cytokines (TNFa/IFNY) excess of glucocorticoids (dexamethasone, Dex) or nutrient deprivation (starvation, PBS). WST formulation resulted able to sustain muscle trophism per se and extremely efficacious in protecting myotubes against MyHC degradation by activating the anabolic pathway, Akt irrespectively from the atrophic stimulus applied. Moreover, WST was able to i) sustain myoblast differentiation by activating p38 MAPK/myogenin axis in presence of Dex and TNFa/IFNy; ii) protect muscle cells against TNFa/IFNy-induced apoptosis; and iii) reduce Dex-dependent activation of the ubiquitin-proteasome system. Phytochemical profiles of the single plant extracts confirmed the presence of multiple metabolites with potential anti-atrophic effects. Furthermore, E. arvense (horsetail), a typical plant of northern Italy recommended as a treatment for osteoporosis [5], emerged from one-hundred medical plant extracts as able to contrast TNFa/IFNy- or Dex-induced muscle atrophy in vitro. Based on our results, low-cost, non-toxic phytotherapy dietary products containing WST and/or $E$. arvense could be developed to maintain muscle mass and functionality in aged people and in diffuse atrophying conditions, thus improving the quality of life and reducing health-care costs.

[1] Dutt V et al., Pharmacol Res. 2015, 46:86-100; [2] Larsson L et al., Physiol Rev. 2019, 99: 427-511; [3] Curtis E et al., J Cell Physiol. 2015, 230:2618-2625; [4] Rondanelli M et al., Evid Based Complement Alternat Med. 2016, 2016: 5970367; [5] Kotwal SD and Badole SR, Indian J Pharmacol. 2016, 48: 312-315

\section{P. 42. Titin gene, transcripts and variants: a challenge for myologists and geneticists Marco Savarese ab, Anna Vihola a,b, Mridul Johari a,b, Per Harald Jonson a,b, Jaakko Sarparanta a,b, Helena Luque a,b, Salla Vallipakka a,b, Sampo Koivunen a,b, Meharji Arumilli a,b, Peter Hackman a,b, Bjarne Udd a,c A Folkhälsan Research Center, Helsinki; s Department of Medical Genetics, Medicum, University of Helsinki; c Department of Neurology, Vaasa Central Hospital, Vaasa, Finland}

Human TTN gene contains 364 exons and encodes titin, a giant protein responsible for the passive elasticity of the muscle. Titin is expressed in both cardiac and skeletal muscle and spans from the $\mathrm{Z}$ disk to the $\mathrm{M}$ line of the sarcomere. Titin transcripts undergo extensive alternative splicing. The theoretical transcript including all putative TTN exons is the inferred complete TTN metatranscript (NM_001267550.1). Shorter isoforms have been identified in adult heart and skeletal muscles. Exons not included in any of the post-natal cardiac and 
skeletal muscle isoforms seem to be expressed during foetal development. The first TTN mutation responsible for a genetic disorder (tibial muscular dystrophy) was identified in 2002. In the last few years, the massive use of high throughput sequencing (HTS) in diagnostics has increased the number of patients diagnosed with a titinopathy. TTN-related diseases have a variable age of onset, progression, and muscle involvement. Heterozygous titin truncating variants, for example, have been associated with dominant dilated cardiomyopathy (DCM), a cardiac disease with reduced penetrance affecting approximately 1 in 250 individuals. The same variants have so far always proved to cause recessive skeletal muscle diseases with a childhood or later onset. The increasing number of diagnosed patients has enabled a preliminary genotypephenotype correlation, showing an inverted relationship between the position of truncation in the M-band and the clinical severity/age of onset of the disease and confirming that a form of congenital arthrogryposis is specifically associated with mutations in exons with a high foetal expression. However, considering its sheer size, its repetitive modular structure, its high number of different, tissue- and developmental stage- specific splicing isoforms, the interpretation of TTN variants remains one of the most significant challenge related to HTS investigation in the field of medical genetics. The identification of the (few) rare causative genetic variants from a large number of clinically irrelevant variations requires an exhaustive workflow, based on an in-depth clinical assessment and a comprehensive DNA, RNA and even protein analysis.

\section{P. 43. High-fat diet increases the risk of environmental heatstroke in mice \\ Matteo Serano a, Antonio Michelucci a, Giorgia Rastelli a, Cecilia Paolini a, Flavia Alessandra Guarnie rb, and Feliciano Protas ía \\ a (CAST, Center for Advanced Studies and Technology; University G. D'Annunzio of Chieti-Pescara, Italy) - b (Department of General Pathology, Londrina State University, Brazil)}

Heat-stroke (HS) is a life-threatening response to heat or physical exertion characterized by an abnormal increase in body temperature $\left(>40^{\circ} \mathrm{C}\right)$ that causes dysfunction of organs, central nervous system and may end with death. Both environmental and exertional HS (EHS), often triggered by a hot and humid environment or by strenuous exercise performed in challenging conditions, are caused by excessive heat production in muscle, which in turn is the result of abnormal $\mathrm{Ca}_{2}+$ leak from the sarcoplasmic reticulum (SR) and oxidative stress. As high fat diet is known to increase oxidative stress, the objective of the present study was to investigate the effects of high-fat diet in the heat-stroke susceptibility of adult wild type (WT) mice (4 months of age). The results collected in this study show that 3 months of high fat diet caused: a) increased heat generation and oxygen consumption during heat stress (assessed by indirect calorimetry); b) elevated oxidative stress in both EDL and Soleus muscles; and c) enhanced sensitivity of isolated EDL muscles to caffeine and temperature during in vitro contracture test (IVCT), the gold standard procedure to verify EHS susceptibility in-vitro. Our data suggest that high-fat diet predispose WT mice to EHS, possibly as a result of increased oxidative stress and release of $\mathrm{Ca}_{2}+$ from SR. This study may have important implications for possible guidelines regarding proper food consumption during periods of intense environmental heat.

\section{P. 44. Intravital 3D bioprinting}

Anna Urciuolo a,b, Ilaria Poli c, Cecilia Laterza d, Luca Brandolino d, Elisa Zambaiti d, Paolo Raffa a, Valentina Scattolini a, Giovanni Giobbe b, Paolo De Coppi b, Laura Brigo d, Stefano Salmaso e, Monica Giomo fand Nicola Elvassore b,d,f

a Institute of Pediatric Research (IRP), Fondazione Città della Speranza, Padova, Italy; b University College London Great Ormond Street Institute of Child Health, London, U;K c ONYEL Biotech srl, Padova, Italy; a Venetian Institute of Molecular Medicine, Padova, Italy; e Department of Pharmaceutical and Pharmacological Science, University of Padova, Italy; ; Department of Industrial Engineering, Italy

$3 \mathrm{D}$ bioprinting aims at replicating the composition, the mechanical properties and the 3D architecture of biological tissues/organs for large spectra of biomedical applications, including the generation of complex in vitro 3D models for drug discovery or biological studies and the development of functional living human constructs suitable for the restoration of tissue/organ function. Despite the tremendous technical advancements in 3D bioprinting, the possibility of fabricating 3D structures into pre-existing 3D environment in vitro or in live animals across tissues has never been reported. We developed new photo-sensitive polymers that allow bio-orthogonal two-photon cycloaddition and crosslinking at wavelength longer than $850 \mathrm{~nm}$ without byproducts. 3D hydrogels with sub-micrometric resolution coupled with 3D positioning and orientation can be 
photo-crosslinked into pre-existing 3D environment in vitro or in vivo across tissues by using deep penetrating near-infrared laser light pulses associated to multiphoton microscopy. Such intravital 3D bioprinting enables the fabrication of complex structures inside tissues of live mice, including the dermis, skeletal muscle and brain. Intravital 3D bioprinting of donor-muscle-derived stem cells under the epimysium of hindlimb muscle in mice leads to the de novo formation of myofibres in the mice. Intravital 3D bioprinting could serve as an in vivo alternative to conventional bioprinting and spatially controlled cell delivery.

\section{P.45. Effects of a 12-week exercise intervention on muscle markers of mitochondrial function in individuals with type 1 diabetes \\ Giosue Annibalini a\#, Dean Minnock b\#, Giacomo Valli ac, Roberta Saltarelli a, Vilberto Stocchi a, Elena Barbieri a,d and Giuseppe De Vito c; \# Equally contributing authors \\ a Department of Biomolecular Sciences, University of Urbino Carlo Bo, Urbino, Italy. s School of Public Health Physiotherapy and Sports Science, University College Dublin, Dublin 4, Ireland. c Department of Biomedical Sciences, University of Padua, Padua, Italy. d Interuniversity Institute of Myology, Urbino, Italy.}

Emerging evidence showed structural, functional and metabolic alterations in the skeletal muscle of people living with type 1 diabetes mellitus (T1DM) [1]. This condition, termed diabetic myopathy, has been associated to a form of accelerated muscle aging as it occurs early in life [2]. However, the underlying mechanisms behind this extremely significant, but often overlooked complication remain to be elucidated. In this study, we compared markers of mitochondrial biogenesis and function between skeletal muscle of T1DM and healthy (CNT) subjects. Moreover, we analysed if the presence of diabetic myopathy might compromise/delay longterm exercise-induced adaptations. Ten sedentary T1DM (4M/6F; $32 \square\} 4$ y) without comorbidities, and ten CNT (4M/6F; 28 $\square 6$ y) were enrolled in the study. Participants partake in a concurrent resistance (20 min; $80 \%$ 1RM) and aerobic (20 min; 80\% HRR) [3] training program (3 times a week) for 12 weeks. Muscle biopsies were collected before (pre) and after (post) training to quantify mitochondrial-related gene expression (PGC1a, MTCO2/COX2 and COX5A); mitochondrial DNA (mtDNA) content; telomere length and the relative level of Mitochondrial Oxidative Phosphorylation System (OXHOPOS) complexes and AMPK phosphorylation. The effect of training on muscle strength (1RM), aerobic capacity $\left(\mathrm{VO}_{2 \max }\right)$ and parameters of glycaemic control (standard deviation (SD) and coefficient of variance \% (CV\%) of interstitial glucose (IG)) was also evaluated. At baseline (pre), the expression of mitochondrially encoded MT-CO2/COX2 mRNA, the mtDNA content, telomere length and the relative level of complex I, III and $V$ of the OXHOPOS complex were lower in T1DM compared to CNT. Pre-training SD and CV\% of IG were higher in T1DM than CNT while baseline muscle strength and $\mathrm{VO}_{2 m a x}$ did not differ. The training program did not ameliorate muscle markers of mitochondrial functioning in T1DM. After the training program, only the CNT showed an increase of $\mathrm{VO}_{2 \text { max }}$ and $1 R M$ increased more in CNT than T1DM. Conversely, the training period leads to better glycaemic control in T1DM and increased AMPK activation in both groups. In conclusion, the reduced adaptation to exercise and decreased mitochondrial functioning markers support the hypothesis of diabetic myopathy and accelerated aging of T1DM muscle. However, exercise improved glycaemic control and partially restored telomeres length in T1DM suggesting that specific exercise training-program might represent a key tool for disease management and delaying the onset of diabetic complications.

[1] Monaco CMF et al, Diabetologia. 2018 Jun;61(6):1411-1423. [2] Monaco CMF et al, Exercise and Sport Sciences Reviews. 2019 Apr;47(2):98-107. [3] Minnock D et al, European Journal of Applied Physiology. 2020 Sep 9. Online ahead of print.

\section{P. 46. Development of the first in man clinical application of autologous mesoangioblasts in mitochondrial myopathy patients \\ Florence van Tienen a,b, Ruby Zelissen a,c, Erika Timmer a,c, Patrick Lindsey a,c, Fong Lin d, Inge Westra d, Pauline Meij d, Janneke Hoeijmakers b,e, Catharina Faber b,, Irenaeus de Coo a,b,f, Hubert Smeets a,b,c}

A Department of Toxicogenomics; s School for Mental Health and Neurosciences (MHeNS); c School for Developmental Biology and Oncology (GROW), Maastricht University Medical Centre+, Maastricht; a Department of Clinical Pharmacy and Toxicology, Leiden University Medical Center, Leiden; e Department of Neurology, Maastricht University Medical Centre+, Maastricht; ${ }_{f}$ Neuromuscular and Mitochondrial research center (NeMo), Rotterdam/Maastricht, The Netherlands 
Mitochondrial myopathy has a large impact on the patients' quality of life and no effective treatment options are currently available. Mesoangioblasts are blood vessel-associated cells that fulfill all requirements to be used as cell therapy medicinal product to combat myopathy and allogeneic mesoangioblast transplantation in mice and dog models of muscular dystrophy resulted in functional improvement. Also, a phase I clinical study in 6 DMD patients demonstrated that allogenic mesoangioblast treatment was relatively safe, but did not result in functional improvement. Major drawback of using donor cells is the requirement for immunosuppressive agents, which can be circumvented by using autologous cells. We demonstrated in multiple carriers of different mitochondrial DNA (mtDNA) point-mutations and large-scale deletion carriers that in half of these patients, their mesoangioblasts contained no or a very low mutation load $(<10 \%)$, despite a much higher mutation load in their skeletal muscle. These mesoangioblasts also displayed normal mitochondrial function, proliferative capacity and myogenic differentiation capacity, demonstrating that these nearly mtDNA-mutation free mesoangioblast can be used for autologous cell therapy. In order to clinically apply autologous mesoangioblasts, we developed and validated the GMP-compliant production protocol to generate sufficient labeled mesoangioblasts, which allows assessing their migration capacity to the muscles. Also, we improved stability of the medicinal product and obtained approval of the Dutch central committee for research involving human subjects to conduct this study. Currently, we are executing this phase I/II clinical study that aims to assess the safety and preliminary efficacy of autologous mesoangioblast treatment.

\section{P. 47. A novel transgenic mouse model for arrhythmogenic cardiomyopathy}

Robin Colpaert a, Muriel Breteau b, Libero Vitiello c, Claudia Sacchetto a,c, Alessandra Lorenzon c,Sandrine Seyen a, Paola Braghetta d, Paolo Bonaldo d, Leon J de Windt a, Paul Oakley b, Alessandra Rampazzo c, Martina Calore a.

a Department of Molecular Genetics, Maastricht University, The Netherlands; s Dolomite Bio, United Kingdom; c Department of Biology, University of Padova; a Department of Molecular Medicine, University of Padova, Italy

Arrhythmogenic cardiomyopathy (ACM) is one of the most commonly inherited cardiomyopathies, characterized by the progressive substitution of the myocardium with (fibro-)fatty tissue. Clinically, ACM is characterized by ventricular arrhythmias, syncope, and sudden cardiac death and shows wide phenotypic heterogeneity. Of the known disease genes, desmosomal proteins plakophilin-2 (PKP2), desmoplakin (DSP), and desmoglein-2 (DSG2) are most commonly mutated. We generated transgenic (Tg) mice overexpressing desmoglein-2 carrying the p.G100R mutation found in an affected patient. These TgG mice present several of the clinical features of ACM, such as fibrous tissue replacement, decreased desmosome size and increased distance between cardiomyocyte membranes intercalated discs. Importantly though, we did not detect the same reductions of the canonical Wnt/b-catenin signalling pathway reported in other ACM models, indicating that additional pathways must be perturbed in this model. To establish the transcriptomic ACM signature at single cell level, cardiomyocytes and non-cardiomyocytes from 3 month-old transgenic and non-transgenic mice were isolated via enzymatic digestion of freshly-isolated ventricles and then encapsulated into droplets with a Nadia Innovate device (Dolomite Bio). Each droplet contained a single cell as well as a bar-coded bead. Quality control analysis indicated that our protocol led to proper sample preparation, which then yielded enough RNA for subsequent sequencing. We described a novel mouse model for ACM recapitulating many features observed in human patients and successfully isolated single adult cardiac cells for RNA sequencing.

\section{P. 48. ASC-exosomes induce a neuroprotective effect on the SOD1(G93A) mouse model of ALS Federica Virlaa, Sylwia Dabrowskaa, Ilaria Scambia, Ermanna Turanoa and Raffaella Mariottia}

a Department of Neurosciences, Biomedicine and Movement Sciences, University of Verona, Italy

Amyotrophic lateral sclerosis (ALS) is a fatal neurodegenerative disorder characterized by progressive motor neuron loss leading to gradual paralysis and death from respiratory failure. Due to the pathogenetic complexity, there are no effective therapies available; however, the use of stem cells represents a promising therapeutic strategy in the treatment of neurodegenerative diseases, including ALS. In particular, their protective effect is exerted by producing soluble factors released through extracellular vesicles. Among these, exosomes can originate from most cell types and play an important role in intercellular communication by the release of their content, especially proteins, miRNA and mRNA. Moreover, thanks to their dimensions, they can easily cross the blood-brain barrier. Our group had already tested and demonstrated that repeated administrations of isolated exosomes from adipose mesenchymal stem cells (ASC-exosomes) delivered by intravenous injections exert a neuroprotective effect on the SOD1(G93A) animals, the most widely used murine model of ALS generated by over expression of mutant allele of human SOD1 gene. To further investigate the beneficial 
effects of ASC-exosomes, in our recent study the administration of ASCexosomes has been tested via intranasal as a different and non-invasive administration route. Especially, a lower and a higher concentration of ASC-exosomes were tested, and administrations were performed starting from the clinical onset until the end stage, every 4 days. The results showed that ASC-exosomes could improve the motor performance of animals, evaluated by specific motor tests; they could also protect lumbar spinal cord motor neurons from neurodegeneration and decreased the astrocytes activation in treated SOD1(G93A) mice. Furthermore, in the peripheral tissues the outcomes showed a higher innervated neuromuscular junctions' number and an attenuated skeletal muscle atrophy in the treated group. Taken all together, these results could provide additional knowledge to better understand the protective effect of exosomes and their promising therapeutic use in neurodegenerative disorders.

\section{P. 49. Muscle types and their Regenerative Potential across Animals}

Letizia Zullo 1,2,", Matteo Bozzo 3, Alon Daya 4, Alessio Di Clemente 1,5, Francesco Paolo Mancini 6, Aram Megighian 7,8, Nir Nesher 4, Eric Rottinger 9, Tal Shomrat 4, Stefano Tiozzo 10, Alberto Zullo 6,* and Simona Candiani 3

1Istituto Italiano di Tecnologia, Center for Micro-BioRobotics \& Center for Synaptic Neuroscience and Technology (NSYN), Genova; 2 IRCCS Ospedale Policlinico San Martino, Genova; з Laboratory of Developmental Neurobiology, Department of Earth, Environment and Life Sciences, University of Genova Italy; 4 Faculty of Marine Sciences, Ruppin Academic Center, Michmoret, Israel; 5 Department of Experimental Medicine, University of Genova; 6 Department of Science and Technology, University of Sannio, Benevento; 7 Department of Biomedical Sciences, University of Padova; 8 Padova Neuroscience Center, University of Padova, Italy; 9 Institute for Research on Cancer and Aging (IRCAN), Université Côte d'Azur, CNRS, INSERM, Nice; 10 Laboratoire de Biologie du Développement de Villefranchesur-

Mer (LBDV), Sorbonne Université, CNRS, Paris, France

Cells with contractile functions are present in almost all metazoans, and so are the related processes of muscle homeostasis and regeneration. Regeneration itself is a complex process unevenly spread across metazoans. In mammals, regenerative capacities are restricted to only a small number of organs yet in other metazoans, the ability to respond to environmental injuries ranges from "simple" wound healing to complete anatomical and functional restoration of the lost or damaged part of the body, including muscles. A particular challenge in regenerative biology concerns the development of reconstructive strategies after muscle-related injuries, but also the treatments of degenerative myopathies for which no reliable clinical strategy exists. By comparing the mechanisms of muscle homeostasis and regeneration throughout the diversity of animal body-plans and life cycles, it is possible to identify conserved and divergent cellular and molecular mechanisms underlying muscle plasticity. Here we wish to provide an overview of muscle regeneration studies in metazoans, highlighting the major regenerative strategies and molecular pathways involved. We emphasize some potential contributions of comparative studies into the biomedical fields, therefore advocating deeper employment of 'non-canonical' animals as models for muscle regeneration studies.

\section{P. 50. Unraveling the extracellular vesicle network to improve stem-cell based regeneration of striated muscles \\ Laura Yedigaryan a, Giorgia Giacomazzi a, Ester Sarra a,b, Nefele Giarratana, Enrico Pozzo, Natacha Breuls and Maurilio Sampaolesi a \\ a Stem Cell Biology and Embryology/Department of Development and Regeneration, KU Leuven, Leuven, Belgium; s Human Anatomy Unit, Department of Public Health, Experimental and Forensic Medicine, University of Pavia, Italy}

Skeletal muscle has the innate ability of growth and regeneration under physiological conditions, and in case of injury upon physical activity or degenerative disorders. It is apparent that chronic muscle illnesses caused by genetic and acquired factors are associated with the complete reshuffling of catabolic and anabolic processes in the muscle niche, leading to structural and functional dysregulation and significant loss in muscle mass. In this scenario, extracellular vesicles (EVs) have been unraveled as hosts of factors capable of altering the state of target cells through the direct action of lipids and proteins or by aiming at the modification of gene expression through messenger RNAs and/or miRNAs (1). Given the significance of miRNAs in post transcriptional gene regulation, enhancing stem cell differentiation may be achieved upon their use (2). In this study, we examined the content of EVs from mouse models of muscular dystrophy, muscular hypertrophy, and 
aged mice. Through the analysis of transcriptomes, proteomes, and miRNAs, signatures of hypertrophic and dystrophic remodeling were unraveled. The implicated effect of EVs derived from the hypertrophic model was confirmed on $\mathrm{C} 2 \mathrm{C} 12$ cells and human mesoangioblasts (MABs) subjected to myogenic differentiation. Since from the RNAseq and proteomics analyses we could not detect good candidates, we focused on the miRNA content of EVs and found specific signatures associated with hypertrophic and dystrophic remodeling. Therefore, we tested several combinations of mimics and antagomirs of identified miRNAs upon the differentiation of hMABs in vitro and in vivo. Overall, we have found that EVs derived from hypertrophic mouse models ameliorate myogenesis both in vitro and in vivo. The implications of the role of EVs in modulating muscle regeneration can affect cell therapy approaches that could be eventually coupled with EV delivery with custom, engineered cargos.

[1] Daniel C. Bittel, Jyoti K. Jaiswal Contribution of Extracellular Vesicles in Rebuilding Injured Muscles. Front Physiol. 2019; 10: 828. [2] Natacha Breuls, Nefele Giarratana, Laura Yedigaryan and Maurilio Sampaolesi, "Epigenetic Modifications in Induced Pluripotent Stem Cells to Boost Myogenic Commitment", in Advances in Stem Cell Biology, volume Induced Pluripotent Stem Cells - Novel Concepts, Editor: Alexander Birbrair, Elsevier, In press.

\section{P. 51. Kca3.1 channels as a tool to protect dystrophic muscle \\ Marta Morotti 1, Valeria Bianconi 2, Stefano Garofalo 1, Chiara Mozzetta 3, Heike Wulff 4, Cristina Limatola 1, Myriam Catalano 1, Francesca Grassi 1 \\ 1Dept. of Physiology and Pharmacology; Sapienza University, Rome; 2 CNR -National Research Council of Italy clo Dept of Biology and Biotechnology "C. Darwin", Sapienza University; з IBPM -Institute of Molecular Biology and Pathology CNR -National Research Council of Italy; 4Dept. of Pharmacology, University of California, Davis, USA}

Pro-inflammatory macrophages (MФs) are constitutively present in the muscles of patients with Duchenne muscular dystrophy (DMD) and release large amounts of nitric oxide (NO), which contributes to muscle damage. Alternatively-activated anti-inflammatory MФs are less harmful, as they reduce NO release by proinflammatory $M \Phi s$ and promote muscle regeneration. In the context of DMD, the main drawback in pushing MФs towards an anti-inflammatory phenotype is that they inhibit apoptosis of activated Fibro- Adipogenic Precursor cells (FAPs), which expand and cause muscle fibrosis. Indeed, in DMD patients, abundance of antiinflammatory $M \Phi$ s correlates with enhanced fibrosis and poor prognosis. However, both macrophage phenotype and fibroblast proliferation critically depend on the activity of $\mathrm{Ca}_{2+-}$ activated $\mathrm{K}+$ channels $\mathrm{KCa} 3.1$ (KCNN4). Blockade of the channels by the selective blocker TRAM-34 enhances the fraction of antiinflammatory $M \Phi s$ in atherosclerotic plaques, while reducing cytotoxic NO production, without affecting phagocytic activity. $\mathrm{KCa} 3.1$ channels are also expressed in fibroblasts and promote their proliferation by amplifying $\mathrm{Ca}_{2}+$ increase induced by TGF $\beta 1$. Thus, acting on $\mathrm{KCa} 3.1$ channels might provide a winning approach in DMD. We are therefore testing the effects of selective channel blocker TRAM-34. In mdx mice of different ages ( 6 to 52 weeks), treatment with TRAM-34 for 4 weeks succeeded in reducing collagen deposits in all the muscles examined (diaphragm, tibialis anterior, quadriceps). At the same time, the percentage of MФs expressing anti-inflammatory markers increased. In vitro experiments using $\mathrm{C} 2 \mathrm{C} 12$ cells show that blockade of KCa3.1 channels does not affect proliferation and differentiation of satellite cells. In summary, our data, although preliminary, support the hypothesis that KCa3.1 channels represent a useful target in the therapy of DMD.

\section{P. 52. Central and peripheral fatigue in Myotonic Dystrophy type 1: proposal of a clinical assessment protocol}

Francesca Torria, Giulia Ricci a, Lucia Chico a, Costanza Simoncini a, Gabriele Siciliano a

a Department of Clinical and Experimental Medicine, University of Pisa, Pisa, Italy

DM1 is an autosomal-dominant muscle disease mainly characterized by muscle weakness and myotonic phenomenon, associated to multisystemic involvement. Muscle weakness and fatigue are frequently described as the main symptoms in DM1 patients, both by scientific literature and in current clinical practice. Among many other factors, oxidative stress has been identified as possibly involved in provoking such symptoms. The aim of this study was to investigate the entity of subjective and physiological fatigue in a 26 DM1 patients (17 males, 9 females, mean age 41.6 years, SD +/- 12.7); subjectively experienced fatigue was studied through Fatigue Severity Scale (FSS), and physiological fatigue was measured with a myometer during an intermittent 
incremental exercise of the forearm muscles; during aerobic exercise, blood samples for oxidative stress balance markers measurement were collected. In our patients, central fatigue worsen during motor contraction; FSS score was significantly correlated to maximal voluntary contraction (before and after the effort, $r$-before = $-0.583, p<0.01, r$-after $=-0.534, p<0.05)$, and to motor disability measured by MRC $(r=-0.496, p<0.05)$; also, the association to lactate baseline reached a nearly significant value $(r=0.378, p=0.057)$. Based on our results and given the frequency of fatigue in this clinical scenario, we propose such exercise training protocol not only for proper clinical management and follow-up of DM1 patients, but also for data collection in DM1 patient registries with the aim of transversally and longitudinally describing such symptoms. 